CRYSTALLOGRAPHIC COMMUNICATIONS

ISSN 2056-9890

Received 16 October 2018

Accepted 19 October 2018

Edited by D.-J. Xu, Zhejiang University (Yuquan Campus), China

Keywords: crystal structure; benzodiazepine; hydrogen bond; $\pi$-stacking; Hirshfeld surface.

CCDC reference: 1874203

Supporting information: this article has supporting information at journals.iucr.org/e

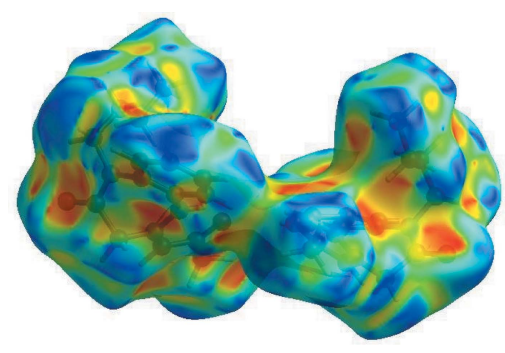

OPEN $\odot$ ACCESS

\section{Crystal structure and Hirshfeld surface analysis of (4Z)-1-butyl-4-(2-oxopropylidene)-2,3,4,5-tetra- hydro-1H-1,5-benzodiazepin-2-one}

\author{
Karim Chkirate, ${ }^{\text {a* Nada Kheira Sebbar, }}{ }^{\mathrm{b}}$ Tuncer Hökelek, ${ }^{\mathrm{c}}$ Damodaran Krishnan, ${ }^{\mathrm{d}}$ \\ Joel T. Mague ${ }^{e}$ and El Mokhtar Essassi ${ }^{a}$
}

\begin{abstract}
aLaboratoire de Chimie Organique Hétérocyclique URAC 21, Pôle de Compétence Pharmacochimie, Av. Ibn Battouta, BP 1014, Faculté des Sciences, Université Mohammed V, Rabat, Morocco, 'baboratoire de Chimie Bioorganique Appliquée, Faculté des sciences, Université Ibn Zohr, Agadir, Morocco, '⿳D Department of Physics, Hacettepe University, 06800 Beytepe, Ankara, Turkey, 'd Department of Chemistry, University of Pittsburgh, Pennsylvania, PA 15260, USA, and ${ }^{\mathbf{e}}$ Department of Chemistry, Tulane University, New Orleans, LA 70118, USA. *Correspondence e-mail: chkiratekarim@gmail.com
\end{abstract}

The asymmetric unit of the title compound, $\mathrm{C}_{16} \mathrm{H}_{20} \mathrm{~N}_{2} \mathrm{O}_{2}$, consists of two independent molecules differing slightly in the conformations of the sevenmembered rings and the butyl substituents, where the benzene rings are oriented at a dihedral angle of $34.56(3)^{\circ}$. In the crystal, pairwise intermolecular $\mathrm{C}-\mathrm{H} \cdots \mathrm{O}$ and complementary intramolecular $\mathrm{C}-\mathrm{H}$. . O hydrogen bonds form twisted strips extending parallel to (012). These strips are connected into layers parallel to (111) by additional intermolecular $\mathrm{C}-\mathrm{H}$. . O hydrogen bonds. The layers are further joined by $\mathrm{C}-\mathrm{H} \cdots \pi$ interactions. The Hirshfeld surface analysis of the crystal structure indicates that the most important contributions for the crystal packing are from $\mathrm{H} \cdots \mathrm{H}(65.5 \%), \mathrm{H} \cdots \mathrm{C} / \mathrm{C} \cdots \mathrm{H}(16.0 \%)$ and $\mathrm{H} \cdots \mathrm{O} / \mathrm{O} \cdots \mathrm{H}(15.8 \%)$ interactions.

\section{Chemical context}

1,5-Benzodiazepine derivatives constituted an important class of heterocyclic compounds possessing a wide spectrum of biological properties. They exhibit anti-inflammatory (Roma et al., 1991), hypnotic (Kudo et al., 1982), anti-HIV-1 (Di Braccio et al., 2001), anticonvulsant (De Sarro et al., 1996), antimicrobial (Kumar et al., 2007) and antitumor (Kamal et al., 2008) activities. The present work is a continuation of the synthesis of the N-substituted 1,5-benzodiazepines derivatives performed recently by our team (Sebhaoui et al., 2016, 2017; Chkirate et al., 2018). In this work, we prepared the title compound, for an investigation of its biological activities, by reacting ( $Z$ )-4-(2-oxopropylidene)-4,5-dihydro- $1 H$-benzo[b][1,5]diazepin-2(3H)-one with 1-bromobutane, under liquidliquid phase-transfer catalysis (PTC) conditions using tetra $n$-butyl ammonium bromide (TBAB) as catalyst and an aqueous solution of potassium hydroxide as base in dichloromethane (Fig. 1). We report herein its crystal and molecular structures along with the Hirshfeld surface analysis.

\section{Structural commentary}

The asymmetric unit of the title compound consists of two independent molecules differing modestly in the conformations of the seven-membered $B(\mathrm{~N} 1 / \mathrm{N} 2 / \mathrm{C} 1 / \mathrm{C} 6-\mathrm{C} 9)$ and $D$ (N3/N4/C17/C22-C25) rings and the $n$-butyl substituents, 
where the benzene $A(\mathrm{C} 1-\mathrm{C} 6)$ and $C(\mathrm{C} 17-\mathrm{C} 22)$ rings are oriented at a dihedral angle of $34.56(3)^{\circ}$. Rings $B$ and $D$ have boat conformations with slightly different Cremer-Pople puckering parameters [for ring $B: Q(2)=0.8872(13) \AA, Q(3)$ $=0.2030(13) \AA, \varphi(2)=28.49(8)^{\circ}$ and $\varphi(3)=138.6(4)^{\circ}, Q_{\mathrm{T}}=$ 0.9102 (13) $\AA$ and for ring $D: Q(2)=0.8631$ (13) $\AA, Q(3)=$ $0.2113(13) \AA, \varphi(2)=24.61(8)^{\circ}$ and $\varphi(3)=136.8(3)^{\circ}, Q_{\mathrm{T}}=$ 0.8886 (13) $\mathrm{]}]$. In the $n$-butyl substituents, the $\mathrm{C} 13-\mathrm{C} 14-$ $\mathrm{C} 15-\mathrm{C} 16 \quad\left[177.96(13)^{\circ}\right]$ and $\mathrm{C} 29-\mathrm{C} 30-\mathrm{C} 31-\mathrm{C} 32$ $\left[174.97(12)^{\circ}\right]$ chains also have slightly different torsion angles. The conformation of the 2-oxopropylidene units are partially determined by the intramolecular $\mathrm{N}-\mathrm{H} \cdots \mathrm{O}$ hydrogen bonds (Table 1, Fig. 1) The r.m.s. deviation of the overlay of two molecules is $0.1367 \AA$.

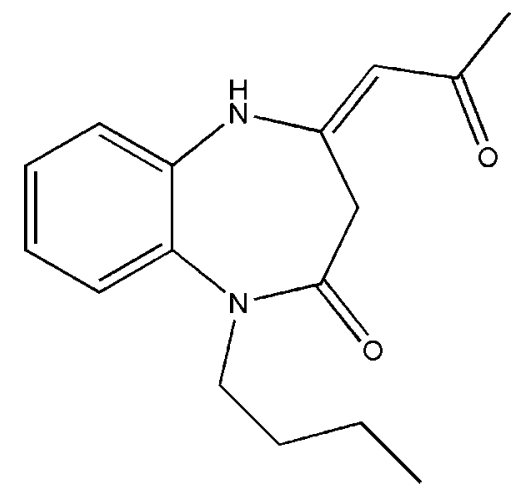

\section{Supramolecular features}

Hydrogen bonding and van der Waals contacts are the dominant interactions in the crystal packing. In the crystal, pairwise intermolecular $\mathrm{C}-\mathrm{H}_{\mathrm{Bnz}} \cdots \mathrm{O}_{\text {Oxoprp }}(\mathrm{Bnz}=$ Benzene and Oxoprp $=2$-oxopropylidene) and complementary intramolecular $\mathrm{C}-\mathrm{H}_{\mathrm{Bnz}} \cdots \mathrm{O}_{\mathrm{Bnzdzp}}($ Bnzdzp $=1,5$-benzodiazepin-2one) hydrogen bonds (Table 1) form twisted strips extending parallel to (012) (Fig. 2). These strips are connected into layers parallel to (111) (Fig. 3) by intermolecular $\mathrm{C}-\mathrm{H}_{\mathrm{Bnz}} \cdots \mathrm{O}_{\text {Oxoprp }}$ and $\mathrm{C}-\mathrm{H}_{\mathrm{Bnzdzp}} \cdots \mathrm{O}_{\mathrm{Bnzdzp}}$ hydrogen bonds (Table 1). The

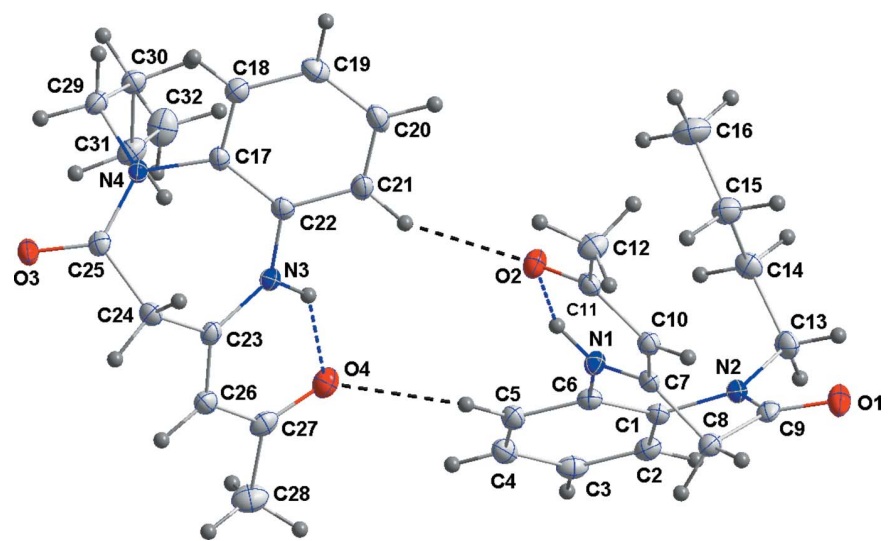

Figure 1

The asymmetric unit with the labelling scheme and $50 \%$ probability ellipsoids. $\mathrm{N}-\mathrm{H} \cdots \mathrm{O}$ and $\mathrm{C}-\mathrm{H} \cdots \mathrm{O}$ hydrogen bonds are indicated by blue and black dashed lines, respectively.

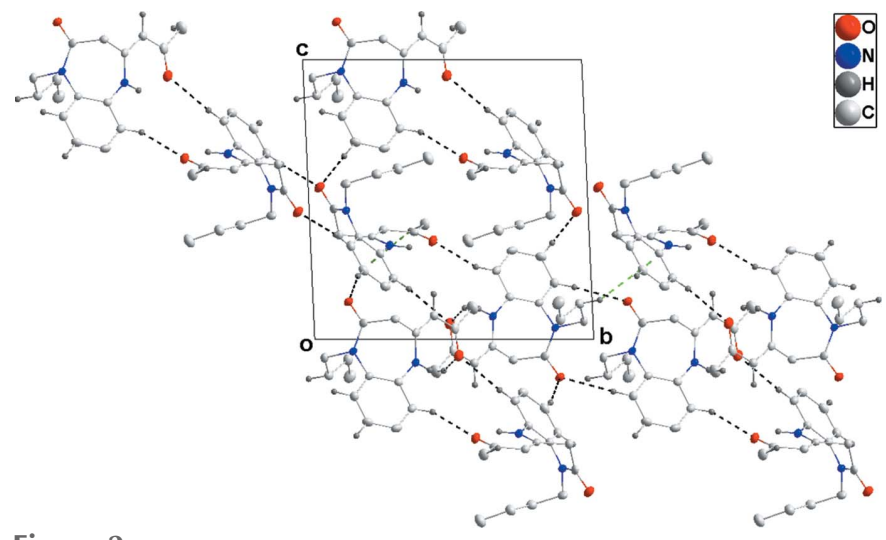

Figure 2

Detail of intermolecular $\mathrm{C}-\mathrm{H} \cdots \mathrm{O}$ hydrogen bonding (black dashed lines) and $\mathrm{C}-\mathrm{H} \cdots \pi$ (ring) interactions (green dashed lines) viewed along the $a$-axis direction.

layers are further joined by $\mathrm{C}-\mathrm{H}_{\mathrm{Bnzdzp}} \cdots \pi$ and $\mathrm{C}-\mathrm{H}_{\mathrm{Bty}} \cdots \pi$ $($ Bty $=n$-butyl) interactions (Table 1 and Figs. 2 and 3).

\section{Hirshfeld surface analysis}

In order to visualize the intermolecular interactions in the crystal of the title compound, a Hirshfeld surface (HS) analysis (Hirshfeld, 1977; Spackman \& Jayatilaka, 2009) was carried out by using Crystal Explorer17.5 (Turner et al., 2017). In the HS plotted over $d_{\text {norm }}$ (Fig. 4), the white surface indicates contacts with distances equal to the sum of van der Waals radii, and the red and blue colours indicate distances shorter (in close contact) or longer (distinct contact) than the van der Waals radii, respectively (Venkatesan et al., 2016). The brightred spots appearing near $\mathrm{O} 1, \mathrm{O} 2, \mathrm{O} 3$ and hydrogen atoms $\mathrm{H} 18, \mathrm{H} 19$ and $\mathrm{H} 28 \mathrm{C}$ indicate their roles as the respective donors and acceptors in the dominant $\mathrm{C}-\mathrm{H} \cdots \mathrm{O}$ and $\mathrm{N}-$ $\mathrm{H}$... O hydrogen bonds. The shape-index of the HS is a tool

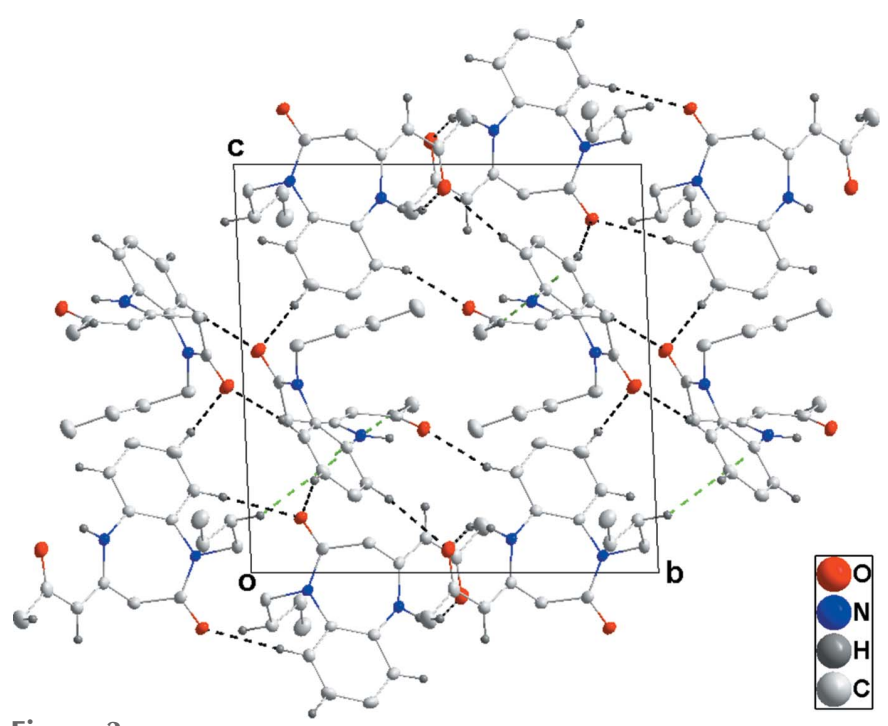

Figure 3

Packing viewed along the $a$-axis direction with intermolecular interactions depicted as in Fig. 2. 
Table 1

Hydrogen-bond geometry $\left(\AA,^{\circ}\right)$.

$C g 1$ is the centroid of benzene ring $A(\mathrm{C} 1-\mathrm{C} 6)$.

\begin{tabular}{lllll}
\hline$D-\mathrm{H} \cdots A$ & $D-\mathrm{H}$ & $\mathrm{H} \cdots A$ & $D \cdots A$ & $D-\mathrm{H} \cdots A$ \\
\hline $\mathrm{N} 1-\mathrm{H} 1 \cdots \mathrm{O} 2$ & $0.927(17)$ & $1.834(17)$ & $2.5998(14)$ & $138.2(13)$ \\
$\mathrm{N} 3-\mathrm{H} 3 A \cdots \mathrm{O} 4$ & $0.898(17)$ & $1.901(17)$ & $2.6349(14)$ & $137.6(14)$ \\
$\mathrm{C} 2-\mathrm{H} 2 \cdots \mathrm{O} 1^{\mathrm{ii}}$ & $0.964(15)$ & $2.469(16)$ & $3.4235(17)$ & $170.6(11)$ \\
$\mathrm{C} 3-\mathrm{H} 3 \cdots 3^{\mathrm{vi}}$ & $0.968(15)$ & $2.420(17)$ & $3.3714(16)$ & $166.0(11)$ \\
$\mathrm{C} 5-\mathrm{H} 5 \cdots \mathrm{O} 4$ & $0.998(16)$ & $2.456(15)$ & $3.4086(17)$ & $159.3(11)$ \\
$\mathrm{C} 18-\mathrm{H} 18 \cdots \mathrm{O} 3^{\mathrm{v}}$ & $0.961(14)$ & $2.556(15)$ & $3.5165(16)$ & $176.4(11)$ \\
$\mathrm{C} 19-\mathrm{H} 19 \cdots 1^{\mathrm{i}}$ & $1.001(15)$ & $2.330(15)$ & $3.3273(15)$ & $177.0(12)$ \\
$\mathrm{C} 21-\mathrm{H} 21 \cdots \mathrm{O} 2$ & $0.986(15)$ & $2.277(15)$ & $3.1933(16)$ & $154.1(11)$ \\
$\mathrm{C} 28-\mathrm{H} 28 C \cdots \mathrm{O} 4^{\text {vi }}$ & 0.98 & 2.48 & $3.4342(18)$ & 164 \\
$\mathrm{C} 12-\mathrm{H} 12 A \cdots C g 1^{\mathrm{x}}$ & $0.999(19)$ & $2.921(19)$ & $3.9047(16)$ & $167.8(13)$ \\
$\mathrm{C} 30-\mathrm{H} 30 A \cdots C g 1^{\mathrm{xii}}$ & $1.007(16)$ & $2.903(15)$ & $3.8016(15)$ & $149.0(11)$
\end{tabular}

Symmetry codes: (i) $-x+1,-y+1,-z+1$; (ii) $\quad-x+2,-y,-z+1$; (v) $-x+1,-y+2,-z$; (vi) $-x+2,-y+1,-z$; (x) $x-1, y, z$; (xii) $x, y+1, z$.

for visualizing $\pi-\pi$ stacking interactions by the presence of adjacent red and blue triangles; if there are no adjacent red and/or blue triangles, then there are no $\pi-\pi$ interactions. Fig. 5 clearly suggests that there are no $\pi-\pi$ interactions.

The overall two-dimensional fingerprint plot, Fig. $6 a$, and those delineated into $\mathrm{H} \cdots \mathrm{H}, \mathrm{H} \cdots \mathrm{C} / \mathrm{C} \cdots \mathrm{H}, \mathrm{H} \cdots \mathrm{O} / \mathrm{O} \cdots \mathrm{H}$, $\mathrm{H} \cdots \mathrm{N} / \mathrm{N} \cdots \mathrm{H}, \mathrm{O} \cdots \mathrm{C} / \mathrm{C} \cdots \mathrm{O}, \mathrm{N} \cdots \mathrm{C} / \mathrm{C} \cdots \mathrm{N}$ and $\mathrm{C} \cdots \mathrm{C}$ contacts (McKinnon et al., 2007) are illustrated in Fig. 6b- $h$, respectively, together with their relative contributions to the Hirshfeld surface. The most important interaction is $\mathrm{H} \cdots \mathrm{H}$ contributing $65.5 \%$ to the overall crystal packing, which is reflected in Fig. $6 b$ as widely scattered points of high density due to the large hydrogen-atom content of the molecule. The wide peak in the centre at $d_{\mathrm{e}}=d_{\mathrm{i}}=1.16 \AA$ in Fig. $6 b$ is due to the short interatomic $\mathrm{H} \cdots \mathrm{H}$ contacts (Table 2). In the presence of weak $\mathrm{C}-\mathrm{H} \cdots \pi$ interactions (Table 1) in the crystal, the pair of characteristic wings resulting in the fingerprint plot delineated into $\mathrm{H} \cdots \mathrm{C} / \mathrm{C} \cdots \mathrm{H}$ contacts, Fig. $6 c$, the $16.0 \%$ contribution to the $\mathrm{HS}$ is viewed as pair of spikes with the tips at $d_{\mathrm{e}}+d_{\mathrm{i}} \sim 2.73 \AA$. The $\mathrm{H} \cdots \mathrm{O} / \mathrm{O} \cdots \mathrm{H}$ contacts in

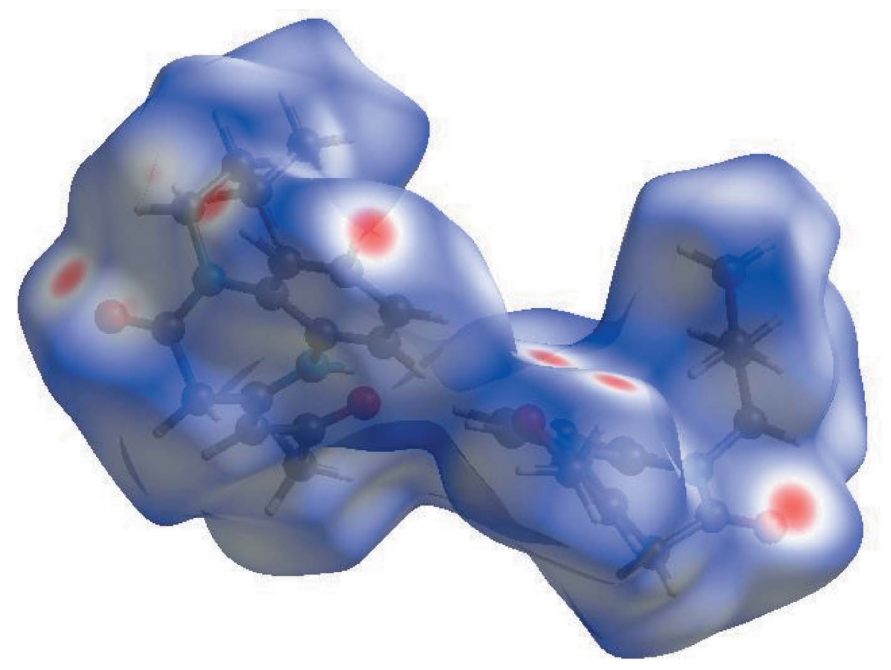

Figure 4

View of the three-dimensional Hirshfeld surface of the title compound plotted over $d_{\text {norm }}$ in the range -0.2745 to 1.3634 a.u.
Table 2

Selected interatomic distances $(\AA)$.

\begin{tabular}{|c|c|c|c|}
\hline $\mathrm{O} 1 \cdots \mathrm{H} 19^{\mathrm{i}}$ & $2.328(16)$ & $\mathrm{C} 11 \cdots \mathrm{H} 26^{\mathrm{iv}}$ & $2.976(14)$ \\
\hline $\mathrm{O} 1 \cdots \mathrm{H} 13 A^{\mathrm{ii}}$ & $2.878(18)$ & $\mathrm{C} 13 \cdots \mathrm{H} 2$ & $2.746(15)$ \\
\hline $\mathrm{O} 1 \cdots \mathrm{H} 2^{\mathrm{ii}}$ & $2.468(15)$ & $\mathrm{C} 17 \cdots \mathrm{H} 24 B$ & $2.635(14)$ \\
\hline $\mathrm{O} 1 \cdots \mathrm{H} 13 B$ & $2.242(15)$ & $\mathrm{C} 17 \cdots \mathrm{H} 30 B$ & $2.810(15)$ \\
\hline $\mathrm{O} 1 \cdots \mathrm{H} 8 B^{\mathrm{iii}}$ & $2.858(15)$ & $\mathrm{C} 18 \cdots \mathrm{H} 29 B$ & 2.688 (14) \\
\hline $\mathrm{O} 2 \cdots \mathrm{H} 21$ & $2.277(15)$ & $\mathrm{C} 21 \cdots \mathrm{H} 14 B^{\mathrm{ix}}$ & $2.961(17)$ \\
\hline $\mathrm{O} 2 \cdots \mathrm{H} 12 C^{\mathrm{i}}$ & $2.627(18)$ & $\mathrm{C} 21 \cdots \mathrm{H} 28 A^{\text {iv }}$ & 2.94 \\
\hline $\mathrm{O} 2 \cdots \mathrm{H} 1$ & $1.834(17)$ & $\mathrm{C} 22 \cdots \mathrm{H} 24 B$ & $2.679(14)$ \\
\hline $\mathrm{O} 2 \cdots \mathrm{H} 26^{\mathrm{iv}}$ & $2.780(14)$ & $\mathrm{C} 25 \cdots \mathrm{H} 29 B^{\mathrm{v}}$ & 2.815 (14) \\
\hline $\mathrm{O} 3 \cdots \mathrm{H} 18^{\mathrm{v}}$ & $2.556(15)$ & $\mathrm{C} 26 \cdots \mathrm{H} 4^{\mathrm{vi}}$ & $2.988(16)$ \\
\hline $\mathrm{O} 3 \cdots \mathrm{H} 3^{\mathrm{vi}}$ & $2.424(15)$ & $\mathrm{C} 27 \cdots \mathrm{H} 3 A$ & 2.459 (17) \\
\hline $\mathrm{O} 3 \cdots \mathrm{H} 29 B^{\mathrm{v}}$ & $2.637(15)$ & 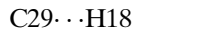 & $2.768(15)$ \\
\hline $\mathrm{O} 3 \cdots \mathrm{H} 29 A$ & $2.300(14)$ & $\mathrm{H} 1 \cdots \mathrm{H} 5$ & $2.48(2)$ \\
\hline $\mathrm{O} 4 \cdots \mathrm{H} 3 A$ & $1.901(18)$ & $\mathrm{H} 2 \cdots \mathrm{H} 13 A$ & $2.26(2)$ \\
\hline $\mathrm{O} 4 \cdots \mathrm{H} 24 B^{\mathrm{iv}}$ & $2.761(14)$ & $\mathrm{H} 2 \cdots \mathrm{H} 13 B^{\mathrm{ii}}$ & $2.51(2)$ \\
\hline $\mathrm{O} 4 \cdots \mathrm{H} 5$ & $2.456(15)$ & $\mathrm{H} 3 A \cdots \mathrm{H} 21$ & $2.39(2)$ \\
\hline $\mathrm{O} 4 \cdots \mathrm{H} 28 C^{\mathrm{vi}}$ & 2.48 & $\mathrm{H} 8 B \cdots \mathrm{H} 10$ & $2.40(2)$ \\
\hline $\mathrm{N} 1 \cdots \mathrm{H} 24 A^{\text {iv }}$ & $2.775(15)$ & $\mathrm{H} 10 \cdots \mathrm{H} 12 A$ & $2.50(2)$ \\
\hline $\mathrm{N} 1 \cdots \mathrm{H} 15 A$ & $2.858(17)$ & $\mathrm{H} 12 A \cdots \mathrm{H} 14 A^{\mathrm{x}}$ & $2.49(2)$ \\
\hline $\mathrm{N} 2 \cdots \mathrm{H} 13 A^{\mathrm{ii}}$ & $2.828(16)$ & $\mathrm{H} 13 B \cdots \mathrm{H} 32 A^{\text {ix }}$ & $2.54(2)$ \\
\hline $\mathrm{N} 2 \cdots \mathrm{H} 15 A$ & $2.704(16)$ & $\mathrm{H} 13 B \cdots \mathrm{H} 15 B$ & $2.57(2)$ \\
\hline $\mathrm{N} 3 \cdots \mathrm{H} 31 B$ & 2.915 (17) & $\mathrm{H} 14 A \cdots \mathrm{H} 16 A$ & $2.52(2)$ \\
\hline $\mathrm{N} 4 \cdots \mathrm{H} 31 B$ & 2.705 (17) & $\mathrm{H} 15 B \cdots \mathrm{H} 32 A^{\mathrm{ix}}$ & $2.54(2)$ \\
\hline $\mathrm{C} 1 \cdots \mathrm{H} 8 A$ & $2.669(16)$ & $\mathrm{H} 18 \cdots \mathrm{H} 29 B$ & $2.21(2)$ \\
\hline $\mathrm{C} 1 \cdots \mathrm{H} 14 A$ & $2.818(15)$ & $\mathrm{H} 24 A \cdots \mathrm{H} 26$ & $2.34(2)$ \\
\hline $\mathrm{C} 2 \cdots \mathrm{H} 13 A$ & $2.790(16)$ & $\mathrm{H} 26 \cdots \mathrm{H} 28 A$ & 2.33 \\
\hline $\mathrm{C} 2 \cdots \mathrm{H} 30 A^{\mathrm{vii}}$ & $2.970(14)$ & $\mathrm{H} 28 B \cdots \mathrm{H} 32 C^{\mathrm{vi}}$ & 2.50 \\
\hline $\mathrm{C} 3 \cdots \mathrm{H} 12 A^{\text {viii }}$ & 2.833 (19) & $\mathrm{H} 29 A \cdots \mathrm{H} 31 A$ & $2.54(2)$ \\
\hline $\mathrm{C} 3 \cdots \mathrm{H} 30 A^{\mathrm{vii}}$ & $2.852(15)$ & $\mathrm{H} 29 A \cdots \mathrm{H} 32 B^{\mathrm{xi}}$ & $2.58(2)$ \\
\hline С6 $\cdots \mathrm{H} 8 A$ & $2.652(15)$ & $\mathrm{H} 30 A \cdots \mathrm{H} 32 B$ & $2.50(2)$ \\
\hline $\mathrm{C} 9 \cdots \mathrm{H} 13 A^{\mathrm{ii}}$ & $2.828(17)$ & $\mathrm{H} 31 A \cdots \mathrm{H} 31 A^{\mathrm{xi}}$ & $2.55(2)$ \\
\hline $\mathrm{C} 10 \cdots \mathrm{H} 20^{\mathrm{i}}$ & $2.871(15)$ & $\mathrm{H} 31 A \cdots \mathrm{H} 32 B^{\mathrm{xi}}$ & $2.57(2)$ \\
\hline $\mathrm{C} 11 \ldots \mathrm{H} 1$ & 2.424 (17) & $\mathrm{H} 32 C \cdots \mathrm{H} 28 B^{\mathrm{vi}}$ & 2.50 \\
\hline
\end{tabular}

Symmetry codes: (i) $-x+1,-y+1,-z+1$; (ii) $\quad-x+2,-y,-z+1$; (iii) $-x+1,-y,-z+1 ; \quad$ (iv) $\quad-x+1,-y+1,-z ; \quad$ (v) $-x+1,-y+2,-z$; (vi) $-x+2,-y+1,-z$; (vii) $x, y-1, z$; (viii) $x+1, y, z$; (ix) $-x+2,-y+1,-z+1$; (x) $x-1, y, z ;(\mathrm{xi})-x+2,-y+2,-z$.

the structure, with $15.8 \%$ contribution to the HS, have a symmetrical distribution of points, Fig. $6 d$, with the tips at $d_{\mathrm{e}}+d_{\mathrm{i}} \sim 2.24 \AA$ arising from the short intra- and/or interatomic $\mathrm{C}-\mathrm{H} \cdots \mathrm{O}$ and $\mathrm{N}-\mathrm{H} \cdots \mathrm{O}$ hydrogen bonding (Table 1) as well as from the $\mathrm{H} \cdots \mathrm{O} / \mathrm{O} \cdots \mathrm{H}$ contacts (Table 2). Finally, the $\mathrm{H} \cdots \mathrm{N} / \mathrm{N} \cdots \mathrm{H}$ (Fig. $6 e$ ) contacts (Table 2) in the structure, with a $1.4 \%$ contribution to the $\mathrm{HS}$, have a symmetrical

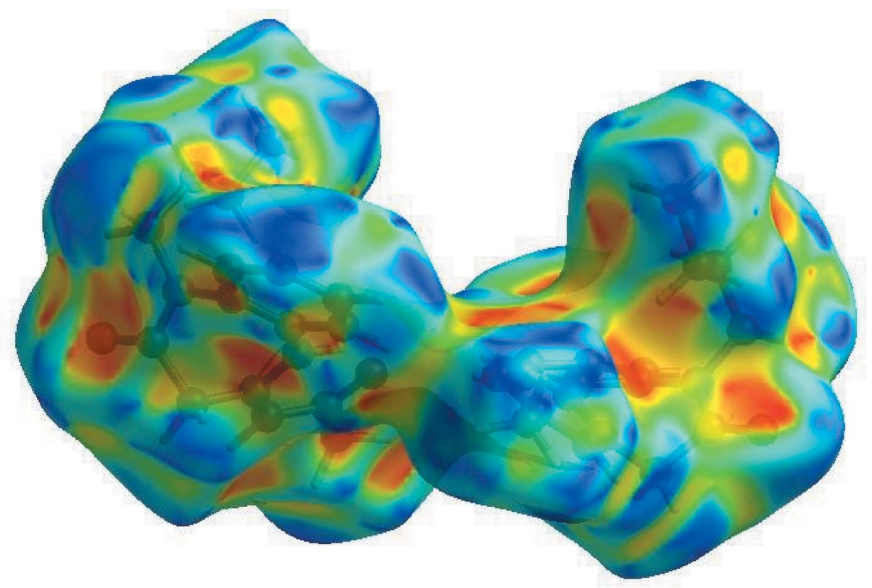

Figure 5

Hirshfeld surface of the title compound plotted over shape-index. 

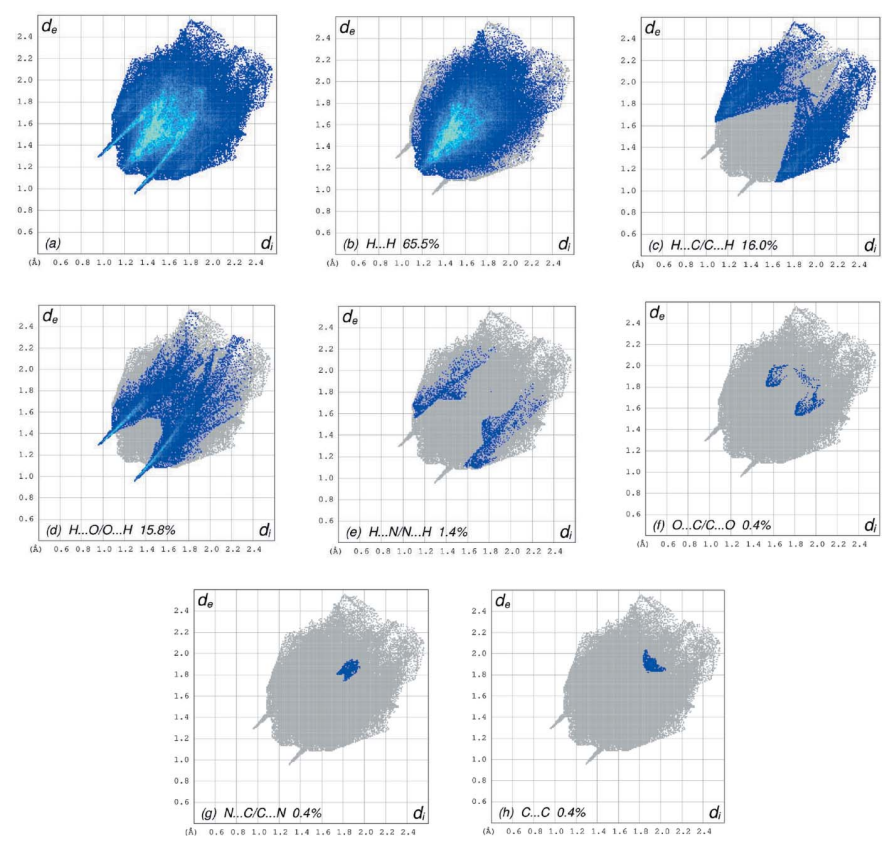

Figure 6

The full two-dimensional fingerprint plots for the title compound, showing (a) all interactions, and delineated into $(b) \mathrm{H} \cdots \mathrm{H},(c) \mathrm{H} \cdots \mathrm{Cl}$ $\mathrm{C} \cdots \mathrm{H},(d) \mathrm{H} \cdots \mathrm{O} / \mathrm{O} \cdots \mathrm{H},(e) \mathrm{H} \cdots \mathrm{N} / \mathrm{N} \cdots \mathrm{H},(f) \mathrm{O} \cdots \mathrm{C} / \mathrm{C} \cdots \mathrm{O},(g) \mathrm{N} \cdots \mathrm{C} /$ $\mathrm{C} \cdots \mathrm{N}$ and $(h) \mathrm{C} \cdots \mathrm{C}$ interactions. $d_{\mathrm{i}}$ and $d_{\mathrm{e}}$ are the closest internal and external distances (in $\AA$ ) from given points on the Hirshfeld surface.

distribution of points, with a pair of wings appearing at $d_{\mathrm{e}}=d_{\mathrm{i}}$ $=2.67 \AA$. The Hirshfeld surface representations for $d_{\text {norm }}$ are shown for the $\mathrm{H} \cdots \mathrm{H}, \mathrm{H} \cdots \mathrm{C} / \mathrm{C} \cdot \mathrm{H}$ and $\mathrm{H} \cdots \mathrm{O} / \mathrm{O} \cdots \mathrm{H}$ interactions in Fig. $7 a-c$, respectively.

The Hirshfeld surface analysis confirms the importance of $\mathrm{H}$-atom contacts in establishing the packing. The large number of $\mathrm{H} \cdots \mathrm{H}, \mathrm{H} \cdots \mathrm{C} / \mathrm{C} \cdots \mathrm{H}$ and $\mathrm{H} \cdots \mathrm{O} / \mathrm{O} \cdots \mathrm{H}$ interactions suggest that van der Waals interactions and hydrogen bonding play the major roles in the crystal packing (Hathwar et al., 2015).

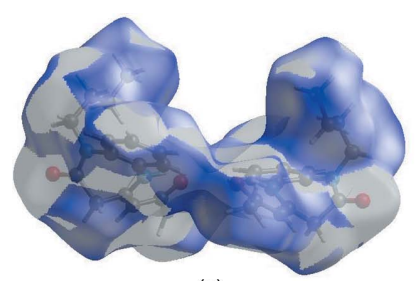

(a)

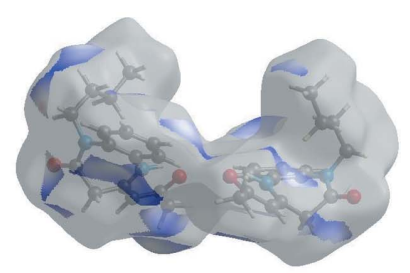

(b)

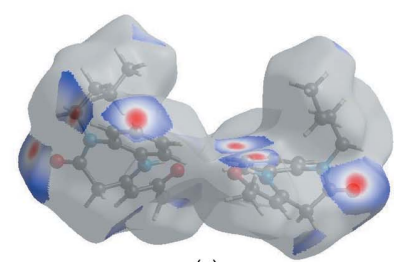

(c)

Figure 7

Hirshfeld surface representations of $d_{\text {norm }}$ for $(a) \mathrm{H} \cdots \mathrm{H},(b) \mathrm{H} \cdots \mathrm{C}$ / $\mathrm{C} \cdot \mathrm{H}$ and $(c) \mathrm{H} \cdots \mathrm{O} / \mathrm{O} \cdots \mathrm{H}$ interactions.
Table 3

Experimental details.

Crystal data

Chemical formula

$M_{\text {r }}$

Crystal system, space group

Temperature (K)

$a, b, c(\AA)$

$\alpha, \beta, \gamma\left(^{\circ}\right)$

$V\left(\AA^{3}\right)$

$Z$

Radiation type

$\mu\left(\mathrm{mm}^{-1}\right)$

Crystal size (mm)

$\mathrm{C}_{16} \mathrm{H}_{20} \mathrm{~N}_{2} \mathrm{O}_{2}$

272.34

Triclinic, $P \overline{1}$

100

9.1132 (6), 12.6676 (9), 12.8164 (9)

91.344 (1), 99.537 (1), 96.340 (1)

1448.87 (17)

4

Mo $K \alpha$

0.08

$0.34 \times 0.29 \times 0.25$

Data collection

Diffractometer

Absorption correction

$T_{\min }, T_{\max }$

No. of measured, independent and observed $[I>2 \sigma(I)]$ reflections

$R_{\text {int }}$

$(\sin \theta / \lambda)_{\max }\left(\AA^{-1}\right)$

Bruker SMART APEX CCD

Multi-scan (TWINABS; Sheldrick, 2009)

$0.97,0.98$

51542, 51542, 40020

0.029

0.696

Refinement

$R\left[F^{2}>2 \sigma\left(F^{2}\right)\right], w R\left(F^{2}\right), S$

No. of reflections

No. of parameters

H-atom treatment

$0.045,0.133,1.08$

51542

511

$\mathrm{H}$ atoms treated by a mixture of independent and constrained refinement

$\Delta \rho_{\max }, \Delta \rho_{\min }\left(\mathrm{e} \AA^{-3}\right)$ $0.41,-0.32$

Computer programs: APEX3 and SAINT (Bruker, 2016), SHELXT (Sheldrick, 2015a), SHELXL2018 (Sheldrick, 2015b), DIAMOND (Brandenburg \& Putz, 2012) and SHELXTL (Sheldrick, 2008).

\section{Synthesis and crystallization}

To a solution of (Z)-4-(2-oxopropylidene)-4,5-dihydro- $1 H$ benzo[b][1,5]diazepin-2 $(3 H)$-one $(2.38 \mathrm{mmol})$ in $15 \mathrm{ml}$ of dichloromethane were added 1.5 eq of 1-bromobutane, $(3.57 \mathrm{mmol})$ of potassium hydroxide dissolved in water and 0.23 mmol of tetra- $n$-butyl ammonium bromide (BTBA). The mixture was kept under magnetic stirring at room temperature for $48 \mathrm{~h}$. A little water was added and then the organic phase was extracted. The mixture obtained was chromatographed on a column of silica gel (eluent hexane/ethyl acetate 8/2) to give three products. The title compound was isolated as the major product in a yield of $77 \%$.

\section{Refinement}

Crystal data, data collection and structure refinement details are summarized in Table 3. $\mathrm{H}$ atoms attached to $\mathrm{C} 28$ did not give a satisfactory geometry so they were positioned geometrically with $\mathrm{C}-\mathrm{H}=0.98 \AA$, and refined as riding with $U_{\text {iso }}(\mathrm{H})$ $=1.5 U_{\text {eq }}(\mathrm{C})$. The remaining $\mathrm{H}$ atoms were located in a difference-Fourier map and were freely refined. The crystal studied was twinned.

\section{Funding information}

JTM thanks Tulane University for support of the Tulane Crystallography Laboratory. TH is grateful to the Hacettepe 
University Scientific Research Project Unit (grant No. 013 D04 602 004).

\section{References}

Brandenburg, K. \& Putz, H. (2012). DIAMOND, Crystal Impact GbR, Bonn, Germany.

Bruker (2016). APEX3, SADABS and SAINT. Bruker AXS Inc., Madison, Wisconsin, USA.

Chkirate, K., Sebbar, N. K., Karrouchi, K. \& Essassi, E. M. (2018). J. Mar. Chim. Heterocycl. 17, 1-27.

De Sarro, G., Gitto, R., Rizzo, M., Zappia, M. \& De Sarro, A. (1996). Gen. Pharmacol. 27, 935-941.

Di Braccio, M., Grossi, G. C., Roma, G., Vargiu, L., Mura, M. \& Marongiu, M. E. (2001). Eur. J. Med. Chem. 36, 935-949.

Hathwar, V. R., Sist, M., Jørgensen, M. R. V., Mamakhel, A. H., Wang, X., Hoffmann, C. M., Sugimoto, K., Overgaard, J. \& Iversen, B. B. (2015). IUCrJ, 2, 563-574.

Hirshfeld, H. L. (1977). Theor. Chim. Acta, 44, 129-138.

Kamal, A., Shankaraiah, N., Prabhakar, S., Reddy, C. R., Markandeya, N., Reddy, K. L. \& Devaiah, X. (2008). Bioorg. Med. Chem. Lett. 18, 2434-2439.
Kudo, Y. (1982). Int. Pharmacopsychiatry, 17, 49-64.

Kumar, R. \& Joshi, Y. C. (2007). Arkivoc. XIII, 142-149.

McKinnon, J. J., Jayatilaka, D. \& Spackman, M. A. (2007). Chem. Commun. 3814-3816.

Roma, G., Grossi, G. C., Di Braccio, M., Ghia, M. \& Mattioli, F. (1991). Eur. J. Med. Chem. 26, 489-496.

Sebhaoui, J., El Bakri, Y., Essassi, E. M. \& Mague, J. T. (2016). IUCrData, 1, x161696.

Sebhaoui, J., El Bakri, Y., Essassi, E. M. \& Mague, J. T. (2017). IUCrData, 2, x171057.

Sheldrick, G. M. (2008). Acta Cryst. A64, 112-122.

Sheldrick, G. M. (2009). TWINABS. University of Göttingen, Göttingen, Germany.

Sheldrick, G. M. (2015a). Acta Cryst. A71, 3-8.

Sheldrick, G. M. (2015b). Acta Cryst. C71, 3-8.

Spackman, M. A. \& Jayatilaka, D. (2009). CrystEngComm, 11, 1932.

Turner, M. J., McKinnon, J. J., Wolff, S. K., Grimwood, D. J., Spackman, P. R., Jayatilaka, D. \& Spackman, M. A. (2017). Crystal. Explorer17. The University of Western Australia.

Venkatesan, P., Thamotharan, S., Ilangovan, A., Liang, H. \& Sundius, T. (2016). Spectrochim. Acta Part A, 153, 625-636. 


\section{supporting information}

Acta Cryst. (2018). E74, 1669-1673 [https://doi.org/10.1107/S2056989018014779]

Crystal structure and Hirshfeld surface analysis of (4Z)-1-butyl-4-(2-oxopropylidene)-2,3,4,5-tetrahydro-1 $H$-1,5-benzodiazepin-2-one

Karim Chkirate, Nada Kheira Sebbar, Tuncer Hökelek, Damodaran Krishnan, Joel T. Mague and El Mokhtar Essassi

Computing details

Data collection: APEX3 (Bruker, 2016); cell refinement: SAINT (Bruker, 2016); data reduction: SAINT; program(s) used to solve structure: SHELXT (Sheldrick, 2015a); program(s) used to refine structure: SHELXL2018 (Sheldrick, 2015b); molecular graphics: DIAMOND (Brandenburg \& Putz, 2012); software used to prepare material for publication: SHELXTL (Sheldrick, 2008).

(4Z)-1-Butyl-4-(2-oxopropylidene)-2,3,4,5-tetrahydro-1H-1,5-benzodiazepin-2-one

Crystal data

$\mathrm{C}_{16} \mathrm{H}_{20} \mathrm{~N}_{2} \mathrm{O}_{2}$

$M_{r}=272.34$

Triclinic, $P \overline{1}$

$a=9.1132(6) \AA$

$b=12.6676(9) \AA$

$c=12.8164(9) \AA$

$\alpha=91.344(1)^{\circ}$

$\beta=99.537(1)^{\circ}$

$\gamma=96.340(1)^{\circ}$

$V=1448.87(17) \AA^{3}$

Data collection

Bruker SMART APEX CCD diffractometer

Radiation source: fine-focus sealed tube Graphite monochromator

Detector resolution: 8.3333 pixels $\mathrm{mm}^{-1}$

$\varphi$ and $\omega$ scans

Absorption correction: multi-scan

(TWINABS; Sheldrick, 2009)

$T_{\min }=0.97, T_{\max }=0.98$

Refinement

Refinement on $F^{2}$

Least-squares matrix: full

$R\left[F^{2}>2 \sigma\left(F^{2}\right)\right]=0.045$

$w R\left(F^{2}\right)=0.133$

$S=1.08$

51542 reflections
$Z=4$

$F(000)=584$

$D_{\mathrm{x}}=1.249 \mathrm{Mg} \mathrm{m}^{-3}$

Mo $K \alpha$ radiation, $\lambda=0.71073 \AA$

Cell parameters from 9901 reflections

$\theta=2.2-29.6^{\circ}$

$\mu=0.08 \mathrm{~mm}^{-1}$

$T=100 \mathrm{~K}$

Block, colourless

$0.34 \times 0.28 \times 0.25 \mathrm{~mm}$

51542 measured reflections

51542 independent reflections

40020 reflections with $I>2 \sigma(I)$

$R_{\text {int }}=0.029$

$\theta_{\max }=29.7^{\circ}, \theta_{\min }=1.6^{\circ}$

$h=-12 \rightarrow 12$

$k=-17 \rightarrow 17$

$l=-17 \rightarrow 17$

511 parameters

0 restraints

Primary atom site location: structure-invariant direct methods

Secondary atom site location: difference Fourier map 
Hydrogen site location: mixed

$\mathrm{H}$ atoms treated by a mixture of independent and constrained refinement

$$
\begin{aligned}
& w=1 /\left[\sigma^{2}\left(F_{\mathrm{o}}^{2}\right)+(0.0752 P)^{2}+0.0382 P\right] \\
& \text { where } P=\left(F_{\mathrm{o}}^{2}+2 F_{\mathrm{c}}^{2}\right) / 3 \\
& (\Delta / \sigma)_{\max }=0.001 \\
& \Delta \rho_{\max }=0.41 \mathrm{e} \AA^{-3} \\
& \Delta \rho_{\min }=-0.32 \mathrm{e} \AA^{-3}
\end{aligned}
$$

Special details

Experimental. The diffraction data were obtained from 3 sets of 400 frames, each of width $0.5^{\circ}$ in $\omega$, colllected at $\varphi=$ $0.00,90.00$ and $180.00^{\circ}$ and 2 sets of 800 frames, each of width $0.45^{\circ}$ in $\varphi$, collected at $\omega=-30.00$ and $210.00^{\circ}$. The scan time was $20 \mathrm{sec} /$ frame. Analysis of 641 reflections having $\mathrm{I} / \sigma(\mathrm{I})>13$ and chosen from the full data set with CELL_NOW (Sheldrick, 2008) showed the crystal to belong to the triclinic system and to be twinned by a $180^{\circ}$ rotation about the reciprocal axis [111]. The raw data were processed using the multi-component version of SAINT under control of the two-component orientation file generated by $C E L L \_N O W$.

Geometry. All esds (except the esd in the dihedral angle between two 1.s. planes) are estimated using the full covariance matrix. The cell esds are taken into account individually in the estimation of esds in distances, angles and torsion angles; correlations between esds in cell parameters are only used when they are defined by crystal symmetry. An approximate (isotropic) treatment of cell esds is used for estimating esds involving l.s. planes.

Refinement. Refinement of $\mathrm{F}^{2}$ against ALL reflections. The weighted R-factor $\mathrm{wR}$ and goodness of fit $\mathrm{S}$ are based on $\mathrm{F}^{2}$, conventional R-factors $\mathrm{R}$ are based on $\mathrm{F}$, with $\mathrm{F}$ set to zero for negative $\mathrm{F}^{2}$. The threshold expression of $\mathrm{F}^{2}>2 \operatorname{sigma}\left(\mathrm{F}^{2}\right)$ is used only for calculating R-factors(gt) etc. and is not relevant to the choice of reflections for refinement. R-factors based

\begin{tabular}{|c|c|c|c|c|}
\hline & $x$ & $y$ & $z$ & $U_{\text {iso }} * / U_{\text {eq }}$ \\
\hline $\mathrm{O} 1$ & $0.72177(11)$ & $0.04051(8)$ & $0.54430(7)$ & $0.0238(2)$ \\
\hline $\mathrm{O} 2$ & $0.56968(10)$ & $0.43831(7)$ & $0.35257(7)$ & $0.0215(2)$ \\
\hline N1 & $0.72935(12)$ & $0.28280(9)$ & $0.33604(8)$ & $0.0158(2)$ \\
\hline $\mathrm{H} 1$ & $0.7127(18)$ & $0.3534(13)$ & $0.3296(12)$ & $0.032(4)^{*}$ \\
\hline N2 & $0.89478(12)$ & $0.13227(8)$ & $0.46108(8)$ & $0.0165(2)$ \\
\hline $\mathrm{C} 1$ & $0.93805(14)$ & $0.17354(10)$ & $0.36609(9)$ & $0.0157(3)$ \\
\hline $\mathrm{C} 2$ & $1.06770(15)$ & $0.14365(11)$ & $0.33411(10)$ & 0.0185 \\
\hline $\mathrm{H} 2$ & $1.1192(17)$ & 0.0914 (12) & $0.3745(11)$ & $0.023(4)^{*}$ \\
\hline $\mathrm{C} 3$ & $1.11875(15)$ & $0.18668(11)$ & $0.24671(11)$ & $0.0204(3)$ \\
\hline H3 & 1.2087 (17) & $0.1646(11)$ & $0.2263(11)$ & $0.023(4)^{*}$ \\
\hline $\mathrm{C} 4$ & $1.04086(15)$ & 0.26135 (11) & 0.18968 (11) & 0.0208 \\
\hline $\mathrm{H} 4$ & $1.0748(17)$ & 0.2907 (12) & $0.1285(12)$ & $0.023(4)^{*}$ \\
\hline $\mathrm{C} 5$ & $0.91218(15)$ & $0.29138(11)$ & $0.21946(10)$ & 0.0185 \\
\hline H5 & $0.8544(18)$ & $0.3453(12)$ & $0.1809(12)$ & $0.026(4)^{*}$ \\
\hline C6 & $0.85894(14)$ & $0.24746(10)$ & $0.30730(9)$ & $0.0155(3)$ \\
\hline $\mathrm{C} 7$ & $0.61586(14)$ & $0.22068(10)$ & $0.36742(9)$ & $0.0151(3)$ \\
\hline $\mathrm{C} 8$ & $0.63211(15)$ & $0.10416(10)$ & $0.37404(10)$ & $0.0178(3)$ \\
\hline H8A & $0.6596(17)$ & $0.0781(12)$ & $0.3060(11)$ & $0.023(4)^{*}$ \\
\hline H8B & $0.5373(17)$ & $0.0641(12)$ & $0.3867(11)$ & $0.023(4)^{*}$ \\
\hline C9 & $0.75241(15)$ & $0.08784(10)$ & $0.46686(10)$ & $0.0169(3)$ \\
\hline $\mathrm{C} 10$ & $0.49225(14)$ & $0.26264(10)$ & $0.39233(9)$ & $0.0162(3)$ \\
\hline $\mathrm{H} 10$ & $0.4120(17)$ & $0.2170(12)$ & $0.4145(11)$ & $0.023(4)^{*}$ \\
\hline $\mathrm{C} 11$ & $0.47391(14)$ & $0.37296(10)$ & $0.38320(9)$ & $0.0171(3)$ \\
\hline
\end{tabular}
on $\mathrm{F}^{2}$ are statistically about twice as large as those based on F, and R- factors based on ALL data will be even larger. Refined as a 2-component twin. Individual refinement of the $\mathrm{H}$-atoms attached to $\mathrm{C} 28$ did not give a satisfactory geometry so these were included as riding contributions in idealized positions.

Fractional atomic coordinates and isotropic or equivalent isotropic displacement parameters $\left(\hat{A}^{2}\right)$ 


\begin{tabular}{|c|c|c|c|c|}
\hline C12 & $0.33482(16)$ & $0.41213(12)$ & $0.41142(12)$ & $0.0235(3)$ \\
\hline $\mathrm{H} 12 \mathrm{~A}$ & $0.243(2)$ & $0.3606(15)$ & $0.3887(14)$ & $0.046(5)^{*}$ \\
\hline $\mathrm{H} 12 \mathrm{~B}$ & $0.321(2)$ & $0.4819(14)$ & $0.3787(13)$ & $0.038(5)^{*}$ \\
\hline $\mathrm{H} 12 \mathrm{C}$ & $0.348(2)$ & $0.4220(14)$ & $0.4879(14)$ & $0.045(5)^{*}$ \\
\hline $\mathrm{C} 13$ & $1.01107(16)$ & $0.13553(11)$ & $0.55643(10)$ & $0.0214(3)$ \\
\hline H13A & $1.086(2)$ & $0.0890(13)$ & $0.5451(13)$ & $0.038(5)^{*}$ \\
\hline H13B & $0.9588(16)$ & $0.1036(11)$ & $0.6123(11)$ & $0.019(4)^{*}$ \\
\hline $\mathrm{C} 14$ & $1.08024(16)$ & $0.24803(11)$ & $0.59066(11)$ & $0.0214(3)$ \\
\hline H14A & $1.1376(17)$ & $0.2780(12)$ & $0.5385(11)$ & $0.023(4) *$ \\
\hline H14B & $1.1531(19)$ & $0.2442(12)$ & $0.6570(13)$ & $0.031(4)^{*}$ \\
\hline $\mathrm{C} 15$ & $0.96927(17)$ & $0.32465(11)$ & $0.60967(12)$ & $0.0246(3)$ \\
\hline $\mathrm{H} 15 \mathrm{~A}$ & $0.899(2)$ & $0.3314(13)$ & $0.5431(13)$ & $0.038(5)^{*}$ \\
\hline H15B & $0.9065(19)$ & $0.2921(13)$ & $0.6622(13)$ & $0.036(4) *$ \\
\hline $\mathrm{C} 16$ & $1.0457(2)$ & $0.43481(13)$ & $0.64748(14)$ & $0.0331(4)$ \\
\hline H16A & $1.109(2)$ & $0.4651(14)$ & $0.5958(14)$ & $0.045(5)^{*}$ \\
\hline H16B & $1.113(2)$ & $0.4355(15)$ & $0.7181(15)$ & $0.051(5)^{*}$ \\
\hline $\mathrm{H} 16 \mathrm{C}$ & $0.973(2)$ & $0.4854(15)$ & $0.6559(13)$ & $0.044(5)^{*}$ \\
\hline $\mathrm{O} 3$ & $0.58897(11)$ & $0.87290(7)$ & $-0.13509(7)$ & $0.0202(2)$ \\
\hline O4 & $0.79438(11)$ & $0.48603(7)$ & $0.05767(7)$ & $0.0237(2)$ \\
\hline N3 & $0.63297(12)$ & $0.64210(9)$ & $0.08256(8)$ & 0.0159 (2) \\
\hline $\mathrm{H} 3 \mathrm{~A}$ & $0.690(2)$ & $0.5925(14)$ & $0.1094(13)$ & $0.035(5)^{*}$ \\
\hline N4 & $0.58830(12)$ & $0.86428(8)$ & $0.04194(8)$ & $0.0145(2)$ \\
\hline $\mathrm{C} 17$ & $0.54349(14)$ & $0.81037(10)$ & $0.13050(9)$ & $0.0148(3)$ \\
\hline $\mathrm{C} 18$ & $0.47824(15)$ & $0.86709(11)$ & $0.20216(10)$ & $0.0182(3)$ \\
\hline H18 & $0.4569(16)$ & $0.9382(12)$ & $0.1862(11)$ & $0.020(4) *$ \\
\hline C19 & $0.44752(16)$ & $0.82329(11)$ & $0.29503(10)$ & $0.0218(3)$ \\
\hline H19 & $0.4001(18)$ & $0.8658(12)$ & $0.3443(12)$ & $0.028(4)^{*}$ \\
\hline $\mathrm{C} 20$ & $0.48108(16)$ & 0.72094 (11) & $0.31750(10)$ & $0.0216(3)$ \\
\hline $\mathrm{H} 20$ & $0.4601(18)$ & $0.6882(12)$ & $0.3839(12)$ & $0.028(4)^{*}$ \\
\hline $\mathrm{C} 21$ & $0.54102(15)$ & $0.66222(11)$ & $0.24625(10)$ & $0.0189(3)$ \\
\hline $\mathrm{H} 21$ & $0.5639(17)$ & $0.5889(12)$ & $0.2591(11)$ & $0.025(4)^{*}$ \\
\hline $\mathrm{C} 22$ & $0.57102(14)$ & $0.70569(10)$ & $0.15174(9)$ & $0.0152(3)$ \\
\hline $\mathrm{C} 23$ & $0.59693(14)$ & $0.63618(10)$ & $-0.02420(9)$ & $0.0157(3)$ \\
\hline $\mathrm{C} 24$ & $0.48707(15)$ & $0.70842(10)$ & $-0.07315(10)$ & $0.0169(3)$ \\
\hline $\mathrm{H} 24 \mathrm{~A}$ & $0.4620(17)$ & $0.6921(11)$ & $-0.1483(12)$ & $0.022(4)^{*}$ \\
\hline H24B & $0.3985(17)$ & $0.7000(11)$ & $-0.0395(11)$ & $0.018(4)^{*}$ \\
\hline $\mathrm{C} 25$ & $0.55790(13)$ & $0.82264(10)$ & $-0.05945(10)$ & $0.0152(3)$ \\
\hline $\mathrm{C} 26$ & $0.65922(15)$ & $0.56812(10)$ & $-0.08471(10)$ & $0.0180(3)$ \\
\hline $\mathrm{H} 26$ & $0.6285(17)$ & $0.5668(11)$ & $-0.1622(11)$ & $0.020(4)^{*}$ \\
\hline $\mathrm{C} 27$ & $0.76044(14)$ & $0.49577(10)$ & $-0.03993(11)$ & $0.0198(3)$ \\
\hline $\mathrm{C} 28$ & $0.82882(17)$ & $0.42914(12)$ & $-0.11377(12)$ & $0.0292(3)$ \\
\hline $\mathrm{H} 28 \mathrm{~A}$ & 0.773193 & 0.430696 & -0.185929 & $0.044^{*}$ \\
\hline $\mathrm{H} 28 \mathrm{~B}$ & 0.824151 & 0.355681 & -0.091077 & $0.044^{*}$ \\
\hline $\mathrm{H} 28 \mathrm{C}$ & 0.933585 & 0.457712 & -0.112321 & $0.044^{*}$ \\
\hline C29 & $0.68172(15)$ & $0.96788(10)$ & $0.06506(11)$ & $0.0178(3)$ \\
\hline $\mathrm{H} 29 \mathrm{~A}$ & $0.6920(15)$ & $0.9984(11)$ & $-0.0031(11)$ & $0.013(3)^{*}$ \\
\hline $\mathrm{H} 29 \mathrm{~B}$ & $0.6320(16)$ & $1.0178(11)$ & $0.1058(11)$ & $0.018(4)^{*}$ \\
\hline C30 & $0.83516(15)$ & $0.95516(11)$ & $0.12782(11)$ & $0.0189(3)$ \\
\hline
\end{tabular}




\begin{tabular}{lllll} 
H30A & $0.8940(17)$ & $1.0277(12)$ & $0.1407(11)$ & $0.025(4)^{*}$ \\
H30B & $0.8225(17)$ & $0.9296(11)$ & $0.2017(11)$ & $0.021(4)^{*}$ \\
C31 & $0.92392(15)$ & $0.88366(11)$ & $0.07175(12)$ & $0.0223(3)$ \\
H31A & $0.9269(17)$ & $0.9081(12)$ & $-0.0022(12)$ & $0.026(4)^{*}$ \\
H31B & $0.8703(18)$ & $0.8104(13)$ & $0.0616(12)$ & $0.028(4)^{*}$ \\
C32 & $1.08216(17)$ & $0.87977(14)$ & $0.13147(14)$ & $0.0300(3)$ \\
H32A & $1.080(2)$ & $0.8497(14)$ & $0.2012(15)$ & $0.045(5)^{*}$ \\
H32B & $1.1370(19)$ & $0.9530(14)$ & $0.1406(12)$ & $0.034(4)^{*}$ \\
H32C & $1.1392(19)$ & $0.8371(13)$ & $0.0902(13)$ & $0.038(5)^{*}$ \\
\hline
\end{tabular}

Atomic displacement parameters $\left(\AA^{2}\right)$

\begin{tabular}{|c|c|c|c|c|c|c|}
\hline & $U^{11}$ & $U^{22}$ & $U^{33}$ & $U^{12}$ & $U^{13}$ & $U^{23}$ \\
\hline $\mathrm{O} 1$ & $0.0278(5)$ & $0.0238(5)$ & $0.0237(5)$ & $0.0096(4)$ & $0.0103(4)$ & $0.0092(4)$ \\
\hline $\mathrm{O} 2$ & $0.0236(5)$ & $0.0172(5)$ & $0.0250(5)$ & $0.0042(4)$ & $0.0056(4)$ & 0.0058 (4) \\
\hline N1 & $0.0169(5)$ & $0.0140(5)$ & $0.0174(5)$ & 0.0042 (4) & $0.0037(4)$ & $0.0030(4)$ \\
\hline N2 & $0.0200(6)$ & $0.0153(5)$ & $0.0140(5)$ & 0.0031 (4) & 0.0011 & $0.0020(4)$ \\
\hline $\mathrm{C} 1$ & $0.0178(6)$ & $0.0144(6)$ & $0.0138(6)$ & $0.0003(5)$ & $0.0010(5)$ & $-0.0004(5)$ \\
\hline $\mathrm{C} 2$ & $0.0174(6)$ & $0.0151(6)$ & $0.0219(7)$ & $0.0024(5)$ & $-0.0002(5)$ & $-0.0013(5)$ \\
\hline $\mathrm{C} 3$ & $0.0162(6)$ & $0.0208(7)$ & $0.0239(7)$ & $-0.0001(5)$ & $0.0044(5)$ & $-0.0056(5)$ \\
\hline $\mathrm{C} 4$ & $0.0212(7)$ & $0.0220(7)$ & $0.0184(6)$ & $-0.0035(5)$ & $0.0050(5)$ & $-0.0009(5)$ \\
\hline $\mathrm{C} 5$ & $0.0199(6)$ & $0.0176(7)$ & $0.0168(6)$ & $0.0005(5)$ & $0.0006(5)$ & $0.0014(5)$ \\
\hline C6 & $0.0152(6)$ & $0.0149(6)$ & $0.0159(6)$ & $0.0013(5)$ & $0.0019(5)$ & $-0.0015(5)$ \\
\hline $\mathrm{C} 7$ & $0.0176(6)$ & $0.0156(6)$ & $0.0112(5)$ & $0.0020(5)$ & $-0.0006(5)$ & $0.0010(5)$ \\
\hline $\mathrm{C} 8$ & $0.0192(6)$ & $0.0150(6)$ & $0.0192(6)$ & $0.0015(5)$ & $0.0036(5)$ & $0.0008(5)$ \\
\hline C9 & $0.0220(7)$ & $0.0123(6)$ & $0.0181(6)$ & $0.0065(5)$ & $0.0056(5)$ & $0.0006(5)$ \\
\hline $\mathrm{C} 10$ & $0.0164(6)$ & $0.0167(6)$ & $0.0150(6)$ & $0.0015(5)$ & $0.0015(5)$ & $0.0014(5)$ \\
\hline C11 & $0.0192(6)$ & $0.0196(7)$ & $0.0123(6)$ & $0.0040(5)$ & $0.0007(5)$ & $0.0009(5)$ \\
\hline $\mathrm{C} 12$ & $0.0220(7)$ & $0.0217(7)$ & $0.0282(8)$ & $0.0067(6)$ & $0.0061(6)$ & $-0.0003(6)$ \\
\hline C13 & $0.0241(7)$ & $0.0225(7)$ & 0.0169 (6) & $0.0073(6)$ & $-0.0019(6)$ & $0.0028(5)$ \\
\hline C14 & $0.0189(7)$ & $0.0242(7)$ & $0.0198(7)$ & $0.0021(5)$ & $-0.0001(6)$ & -0.0001 \\
\hline $\mathrm{C} 15$ & $0.0246(7)$ & $0.0225(7)$ & $0.0254(7)$ & $0.0026(6)$ & $0.0013(6)$ & $-0.0023(6)$ \\
\hline $\mathrm{C} 16$ & $0.0341(9)$ & $0.0256(8)$ & $0.0373(9)$ & $0.0006(7)$ & $0.0024(8)$ & $-0.0083(7)$ \\
\hline $\mathrm{O} 3$ & $0.0272(5)$ & $0.0192(5)$ & $0.0164(4)$ & $0.0063(4)$ & $0.0071(4)$ & $0.0050(4)$ \\
\hline $\mathrm{O} 4$ & $0.0235(5)$ & $0.0206(5)$ & $0.0277(5)$ & $0.0055(4)$ & 0.0039 (4) & $0.0050(4)$ \\
\hline N3 & $0.0187(5)$ & $0.0137(5)$ & $0.0155(5)$ & $0.0040(4)$ & $0.0016(4)$ & $0.0022(4)$ \\
\hline N4 & $0.0161(5)$ & $0.0137(5)$ & $0.0139(5)$ & $0.0013(4)$ & $0.0028(4)$ & $0.0030(4)$ \\
\hline $\mathrm{C} 17$ & $0.0141(6)$ & $0.0161(6)$ & $0.0134(6)$ & $-0.0004(5)$ & $0.0014(5)$ & $0.0028(5)$ \\
\hline $\mathrm{C} 18$ & $0.0182(6)$ & $0.0169(7)$ & $0.0192(6)$ & $0.0017(5)$ & $0.0028(5)$ & $0.0006(5)$ \\
\hline C19 & $0.0222(7)$ & $0.0262(8)$ & $0.0172(6)$ & $0.0012(6)$ & $0.0059(6)$ & $-0.0024(5)$ \\
\hline $\mathrm{C} 20$ & $0.0236(7)$ & $0.0259(7)$ & $0.0141(6)$ & $-0.0027(6)$ & $0.0032(5)$ & $0.0031(5)$ \\
\hline $\mathrm{C} 21$ & $0.0204(7)$ & $0.0178(7)$ & $0.0165(6)$ & $-0.0013(5)$ & $-0.0005(5)$ & $0.0032(5)$ \\
\hline C22 & $0.0134(6)$ & $0.0164(6)$ & $0.0145(6)$ & $0.0001(5)$ & $0.0003(5)$ & $0.0002(5)$ \\
\hline $\mathrm{C} 23$ & $0.0157(6)$ & $0.0131(6)$ & $0.0171(6)$ & $-0.0018(5)$ & $0.0014(5)$ & $0.0025(5)$ \\
\hline $\mathrm{C} 24$ & $0.0171(6)$ & $0.0178(7)$ & $0.0148(6)$ & $0.0017(5)$ & $-0.0002(5)$ & $0.0008(5)$ \\
\hline $\mathrm{C} 25$ & $0.0136(6)$ & $0.0162(6)$ & $0.0168(6)$ & $0.0060(5)$ & $0.0023(5)$ & $0.0027(5)$ \\
\hline C26 & $0.0196(6)$ & $0.0165(6)$ & $0.0174(6)$ & $-0.0010(5)$ & $0.0034(5)$ & $0.0003(5)$ \\
\hline $\mathrm{C} 27$ & $0.0159(6)$ & $0.0154(6)$ & $0.0271(7)$ & $-0.0025(5)$ & $0.0043(5)$ & -0.0005 \\
\hline
\end{tabular}




\begin{tabular}{lllllll} 
C28 & $0.0256(7)$ & $0.0271(8)$ & $0.0362(8)$ & $0.0070(6)$ & $0.0071(6)$ & $-0.0053(6)$ \\
C29 & $0.0202(6)$ & $0.0132(6)$ & $0.0206(6)$ & $0.0013(5)$ & $0.0056(5)$ & $0.0016(5)$ \\
C30 & $0.0185(6)$ & $0.0180(7)$ & $0.0191(6)$ & $-0.0017(5)$ & $0.0024(5)$ & $0.0002(5)$ \\
C31 & $0.0185(7)$ & $0.0195(7)$ & $0.0289(8)$ & $0.0015(5)$ & $0.0047(6)$ & $0.0005(6)$ \\
C32 & $0.0204(7)$ & $0.0333(9)$ & $0.0368(9)$ & $0.0054(7)$ & $0.0036(7)$ & $0.0089(7)$ \\
\hline
\end{tabular}

Geometric parameters $\left(\hat{A},{ }^{\circ}\right)$

\begin{tabular}{|c|c|c|c|}
\hline $\mathrm{O} 1-\mathrm{C} 9$ & $1.2269(14)$ & $\mathrm{O} 3-\mathrm{C} 25$ & $1.2271(14)$ \\
\hline $\mathrm{O} 2-\mathrm{C} 11$ & $1.2532(15)$ & $\mathrm{O} 4-\mathrm{C} 27$ & $1.2503(16)$ \\
\hline $\mathrm{N} 1-\mathrm{C} 7$ & $1.3504(16)$ & $\mathrm{N} 3-\mathrm{C} 23$ & $1.3515(16)$ \\
\hline $\mathrm{N} 1-\mathrm{C} 6$ & $1.4086(15)$ & $\mathrm{N} 3-\mathrm{C} 22$ & $1.4095(15)$ \\
\hline $\mathrm{N} 1-\mathrm{H} 1$ & $0.927(17)$ & $\mathrm{N} 3-\mathrm{H} 3 \mathrm{~A}$ & $0.898(17)$ \\
\hline $\mathrm{N} 2-\mathrm{C} 9$ & $1.3704(16)$ & $\mathrm{N} 4-\mathrm{C} 25$ & $1.3637(15)$ \\
\hline $\mathrm{N} 2-\mathrm{C} 1$ & $1.4333(15)$ & $\mathrm{N} 4-\mathrm{C} 17$ & $1.4316(14)$ \\
\hline $\mathrm{N} 2-\mathrm{C} 13$ & $1.4755(17)$ & $\mathrm{N} 4-\mathrm{C} 29$ & $1.4786(16)$ \\
\hline $\mathrm{C} 1-\mathrm{C} 2$ & $1.3998(17)$ & $\mathrm{C} 17-\mathrm{C} 18$ & $1.3993(17)$ \\
\hline $\mathrm{C} 1-\mathrm{C} 6$ & $1.4023(18)$ & $\mathrm{C} 17-\mathrm{C} 22$ & $1.4011(17)$ \\
\hline $\mathrm{C} 2-\mathrm{C} 3$ & $1.3833(18)$ & $\mathrm{C} 18-\mathrm{C} 19$ & $1.3820(18)$ \\
\hline $\mathrm{C} 2-\mathrm{H} 2$ & $0.964(15)$ & $\mathrm{C} 18-\mathrm{H} 18$ & $0.961(14)$ \\
\hline $\mathrm{C} 3-\mathrm{C} 4$ & $1.3935(19)$ & $\mathrm{C} 19-\mathrm{C} 20$ & 1.3905 (19) \\
\hline $\mathrm{C} 3-\mathrm{H} 3$ & $0.968(15)$ & C19-H19 & $1.001(15)$ \\
\hline $\mathrm{C} 4-\mathrm{C} 5$ & $1.3804(18)$ & $\mathrm{C} 20-\mathrm{C} 21$ & $1.3816(18)$ \\
\hline $\mathrm{C} 4-\mathrm{H} 4$ & $0.958(14)$ & $\mathrm{C} 20-\mathrm{H} 20$ & $0.993(14)$ \\
\hline $\mathrm{C} 5-\mathrm{C} 6$ & $1.4012(17)$ & $\mathrm{C} 21-\mathrm{C} 22$ & $1.3974(16)$ \\
\hline $\mathrm{C} 5-\mathrm{H} 5$ & $0.998(16)$ & $\mathrm{C} 21-\mathrm{H} 21$ & $0.986(15)$ \\
\hline $\mathrm{C} 7-\mathrm{C} 10$ & $1.3772(17)$ & $\mathrm{C} 23-\mathrm{C} 26$ & $1.3746(17)$ \\
\hline $\mathrm{C} 7-\mathrm{C} 8$ & $1.5025(17)$ & $\mathrm{C} 23-\mathrm{C} 24$ & $1.5014(17)$ \\
\hline $\mathrm{C} 8-\mathrm{C} 9$ & $1.5131(19)$ & $\mathrm{C} 24-\mathrm{C} 25$ & $1.5118(18)$ \\
\hline $\mathrm{C} 8-\mathrm{H} 8 \mathrm{~A}$ & $1.005(14)$ & $\mathrm{C} 24-\mathrm{H} 24 \mathrm{~A}$ & $0.964(15)$ \\
\hline $\mathrm{C} 8-\mathrm{H} 8 \mathrm{~B}$ & $0.992(15)$ & $\mathrm{C} 24-\mathrm{H} 24 \mathrm{~B}$ & $0.974(14)$ \\
\hline $\mathrm{C} 10-\mathrm{C} 11$ & $1.4309(17)$ & $\mathrm{C} 26-\mathrm{C} 27$ & $1.4339(18)$ \\
\hline $\mathrm{C} 10-\mathrm{H} 10$ & $0.966(15)$ & $\mathrm{C} 26-\mathrm{H} 26$ & $0.986(14)$ \\
\hline $\mathrm{C} 11-\mathrm{C} 12$ & $1.5054(18)$ & $\mathrm{C} 27-\mathrm{C} 28$ & $1.5068(18)$ \\
\hline $\mathrm{C} 12-\mathrm{H} 12 \mathrm{~A}$ & $0.999(19)$ & $\mathrm{C} 28-\mathrm{H} 28 \mathrm{~A}$ & 0.9800 \\
\hline $\mathrm{C} 12-\mathrm{H} 12 \mathrm{~B}$ & 0.999 (17) & $\mathrm{C} 28-\mathrm{H} 28 \mathrm{~B}$ & 0.9800 \\
\hline $\mathrm{C} 12-\mathrm{H} 12 \mathrm{C}$ & $0.971(18)$ & $\mathrm{C} 28-\mathrm{H} 28 \mathrm{C}$ & 0.9800 \\
\hline $\mathrm{C} 13-\mathrm{C} 14$ & $1.5170(19)$ & $\mathrm{C} 29-\mathrm{C} 30$ & $1.5203(19)$ \\
\hline $\mathrm{C} 13-\mathrm{H} 13 \mathrm{~A}$ & $0.979(17)$ & $\mathrm{C} 29-\mathrm{H} 29 \mathrm{~A}$ & $0.977(13)$ \\
\hline $\mathrm{C} 13-\mathrm{H} 13 \mathrm{~B}$ & $0.993(13)$ & $\mathrm{C} 29-\mathrm{H} 29 \mathrm{~B}$ & $1.002(14)$ \\
\hline $\mathrm{C} 14-\mathrm{C} 15$ & $1.5204(19)$ & $\mathrm{C} 30-\mathrm{C} 31$ & $1.5215(18)$ \\
\hline $\mathrm{C} 14-\mathrm{H} 14 \mathrm{~A}$ & $0.974(14)$ & $\mathrm{C} 30-\mathrm{H} 30 \mathrm{~A}$ & $1.007(16)$ \\
\hline $\mathrm{C} 14-\mathrm{H} 14 \mathrm{~B}$ & 0.994 (17) & $\mathrm{C} 30-\mathrm{H} 30 \mathrm{~B}$ & $1.028(14)$ \\
\hline $\mathrm{C} 15-\mathrm{C} 16$ & $1.520(2)$ & $\mathrm{C} 31-\mathrm{C} 32$ & $1.523(2)$ \\
\hline C15-H15A & $0.990(18)$ & $\mathrm{C} 31-\mathrm{H} 31 \mathrm{~A}$ & $1.008(14)$ \\
\hline C15-H15B & $1.020(16)$ & $\mathrm{C} 31-\mathrm{H} 31 \mathrm{~B}$ & 0.995 (16) \\
\hline C16-H16A & 1.005 (18) & $\mathrm{C} 32-\mathrm{H} 32 \mathrm{~A}$ & $0.982(17)$ \\
\hline C16-H16B & $1.00(2)$ & $\mathrm{C} 32-\mathrm{H} 32 \mathrm{~B}$ & $0.998(18)$ \\
\hline
\end{tabular}




\begin{tabular}{|c|c|c|c|}
\hline $\mathrm{C} 16-\mathrm{H} 16 \mathrm{C}$ & $0.986(19)$ & $\mathrm{C} 32-\mathrm{H} 32 \mathrm{C}$ & $0.990(17)$ \\
\hline $\mathrm{O} 1 \cdots \mathrm{H} 19^{\mathrm{i}}$ & $2.328(16)$ & $\mathrm{C} 11 \cdots \mathrm{H} 26^{\mathrm{iv}}$ & $2.976(14)$ \\
\hline $\mathrm{O} 1 \cdots \mathrm{H} 13 \mathrm{~A}^{\mathrm{ii}}$ & $2.878(18)$ & $\mathrm{C} 13 \cdots \mathrm{H} 2$ & $2.746(15)$ \\
\hline $\mathrm{O} 1 \cdots \mathrm{H} 2^{\mathrm{ii}}$ & $2.468(15)$ & $\mathrm{C} 17 \cdots \mathrm{H} 24 \mathrm{~B}$ & $2.635(14)$ \\
\hline $\mathrm{O} 1 \cdots \mathrm{H} 13 \mathrm{~B}$ & $2.242(15)$ & $\mathrm{C} 17 \cdots \mathrm{H} 30 \mathrm{~B}$ & $2.810(15)$ \\
\hline $\mathrm{O} 1 \cdots \mathrm{H} 8 \mathrm{~B}^{\mathrm{iii}}$ & $2.858(15)$ & $\mathrm{C} 18 \cdots \mathrm{H} 29 \mathrm{~B}$ & $2.688(14)$ \\
\hline $\mathrm{O} 2 \cdots \mathrm{H} 21$ & $2.277(15)$ & $\mathrm{C} 21 \cdots \mathrm{H} 14 \mathrm{~B}^{\mathrm{ix}}$ & $2.961(17)$ \\
\hline $\mathrm{O} 2 \cdots \mathrm{H} 12 \mathrm{C}^{\mathrm{i}}$ & $2.627(18)$ & $\mathrm{C} 21 \cdots \mathrm{H} 28 \mathrm{~A}^{\mathrm{iv}}$ & 2.94 \\
\hline $\mathrm{O} 2 \cdots \mathrm{H} 1$ & $1.834(17)$ & $\mathrm{C} 22 \cdots \mathrm{H} 24 \mathrm{~B}$ & $2.679(14)$ \\
\hline $\mathrm{O} 2 \cdots \mathrm{H} 26^{\mathrm{iv}}$ & $2.780(14)$ & $\mathrm{C} 25 \cdots \mathrm{H} 29 \mathrm{~B}^{\mathrm{v}}$ & $2.815(14)$ \\
\hline $\mathrm{O} 3 \cdots \mathrm{H} 18^{\mathrm{v}}$ & $2.556(15)$ & $\mathrm{C} 26 \cdots \mathrm{H} 4^{\mathrm{vi}}$ & $2.988(16)$ \\
\hline $\mathrm{O} 3 \cdots \mathrm{H} 3^{\mathrm{vi}}$ & $2.424(15)$ & $\mathrm{C} 27 \cdots \mathrm{H} 3 \mathrm{~A}$ & 2.459 (17) \\
\hline $\mathrm{O} 3 \cdots \mathrm{H} 29 \mathrm{~B}^{\mathrm{v}}$ & $2.637(15)$ & $\mathrm{C} 29 \cdots \mathrm{H} 18$ & $2.768(15)$ \\
\hline $\mathrm{O} 3 \cdots \mathrm{H} 29 \mathrm{~A}$ & $2.300(14)$ & $\mathrm{H} 1 \cdots \mathrm{H} 5$ & $2.48(2)$ \\
\hline $\mathrm{O} 4 \cdots \mathrm{H} 3 \mathrm{~A}$ & $1.901(18)$ & 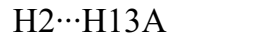 & $2.26(2)$ \\
\hline $\mathrm{O} 4 \cdots \mathrm{H} 24 \mathrm{~B}^{\mathrm{iv}}$ & $2.761(14)$ & $\mathrm{H} 2 \cdots \mathrm{H} 13 \mathrm{~B}^{\mathrm{ii}}$ & $2.51(2)$ \\
\hline $\mathrm{O} 4 \cdots \mathrm{H} 5$ & $2.456(15)$ & $\mathrm{H} 3 \mathrm{~A} \cdots \mathrm{H} 21$ & $2.39(2)$ \\
\hline $\mathrm{O} 4 \cdots \mathrm{H} 28 \mathrm{C}^{\mathrm{vi}}$ & 2.48 & H8B $\cdots \mathrm{H} 10$ & $2.40(2)$ \\
\hline $\mathrm{N} 1 \cdots \mathrm{H} 24 \mathrm{~A}^{\mathrm{iv}}$ & $2.775(15)$ & 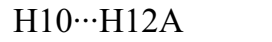 & $2.50(2)$ \\
\hline $\mathrm{N} 1 \cdots \mathrm{H} 15 \mathrm{~A}$ & $2.858(17)$ & $\mathrm{H} 12 \mathrm{~A} \cdots \mathrm{H} 14 \mathrm{~A}^{\mathrm{x}}$ & $2.49(2)$ \\
\hline $\mathrm{N} 2 \cdots \mathrm{H} 13 \mathrm{~A}^{\mathrm{ii}}$ & $2.828(16)$ & $\mathrm{H} 13 \mathrm{~B} \cdots \mathrm{H} 32 \mathrm{~A}^{\mathrm{ix}}$ & $2.54(2)$ \\
\hline $\mathrm{N} 2 \cdots \mathrm{H} 15 \mathrm{~A}$ & 2.704 (16) & H13B $\cdots H 15 B$ & $2.57(2)$ \\
\hline 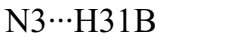 & $2.915(17)$ & H14A $\cdots H 16 A$ & $2.52(2)$ \\
\hline $\mathrm{N} 4 \cdots \mathrm{H} 31 \mathrm{~B}$ & $2.705(17)$ & $\mathrm{H} 15 \mathrm{~B} \cdots \mathrm{H} 32 \mathrm{~A}^{\mathrm{ix}}$ & $2.54(2)$ \\
\hline $\mathrm{C} 1 \cdots \mathrm{H} 8 \mathrm{~A}$ & $2.669(16)$ & $\mathrm{H} 18 \cdots \mathrm{H} 29 \mathrm{~B}$ & $2.21(2)$ \\
\hline $\mathrm{C} 1 \cdots \mathrm{H} 14 \mathrm{~A}$ & $2.818(15)$ & $\mathrm{H} 24 \mathrm{~A} \cdots \mathrm{H} 26$ & $2.34(2)$ \\
\hline $\mathrm{C} 2 \cdots \mathrm{H} 13 \mathrm{~A}$ & $2.790(16)$ & $\mathrm{H} 26 \cdots \mathrm{H} 28 \mathrm{~A}$ & 2.33 \\
\hline $\mathrm{C} 2 \cdots \mathrm{H}_{3} 0 \mathrm{~A}^{\mathrm{vii}}$ & $2.970(14)$ & $\mathrm{H} 28 \mathrm{~B} \cdots \mathrm{H} 32 \mathrm{C}^{\mathrm{vi}}$ & 2.50 \\
\hline $\mathrm{C} 3 \cdots \mathrm{H} 12 \mathrm{~A}^{\text {viii }}$ & 2.833 (19) & H29A $\cdots H 31$ A & $2.54(2)$ \\
\hline $\mathrm{C} 3 \cdots \mathrm{H}_{3} 0 \mathrm{~A}^{\mathrm{vii}}$ & $2.852(15)$ & $\mathrm{H} 29 \mathrm{~A} \cdots \mathrm{H} 32 \mathrm{~B}^{\mathrm{xi}}$ & $2.58(2)$ \\
\hline C6 $\cdots \mathrm{H} 8 \mathrm{~A}$ & $2.652(15)$ & Н30A $\cdots$ H32B & $2.50(2)$ \\
\hline $\mathrm{C} 9 \cdots \mathrm{H} 13 \mathrm{~A}^{\mathrm{ii}}$ & $2.828(17)$ & $\mathrm{H} 31 \mathrm{~A} \cdots \mathrm{H} 31 \mathrm{~A}^{\mathrm{xi}}$ & $2.55(2)$ \\
\hline $\mathrm{C} 10 \cdots \mathrm{H} 20^{\mathrm{i}}$ & $2.871(15)$ & $\mathrm{H} 31 \mathrm{~A} \cdots \mathrm{H} 32 \mathrm{~B}^{\mathrm{xi}}$ & $2.57(2)$ \\
\hline $\mathrm{C} 11 \cdots \mathrm{H} 1$ & $2.424(17)$ & $\mathrm{H} 32 \mathrm{C} \cdots \mathrm{H} 28 \mathrm{~B}^{\mathrm{vi}}$ & 2.50 \\
\hline $\mathrm{C} 7-\mathrm{N} 1-\mathrm{C} 6$ & $125.71(11)$ & $\mathrm{C} 23-\mathrm{N} 3-\mathrm{C} 22$ & $125.86(11)$ \\
\hline $\mathrm{C} 7-\mathrm{N} 1-\mathrm{H} 1$ & $113.8(10)$ & $\mathrm{C} 23-\mathrm{N} 3-\mathrm{H} 3 \mathrm{~A}$ & $114.1(10)$ \\
\hline $\mathrm{C} 6-\mathrm{N} 1-\mathrm{H} 1$ & $120.3(10)$ & $\mathrm{C} 22-\mathrm{N} 3-\mathrm{H} 3 \mathrm{~A}$ & $119.2(10)$ \\
\hline $\mathrm{C} 9-\mathrm{N} 2-\mathrm{C} 1$ & $123.36(10)$ & $\mathrm{C} 25-\mathrm{N} 4-\mathrm{C} 17$ & $123.89(10)$ \\
\hline $\mathrm{C} 9-\mathrm{N} 2-\mathrm{C} 13$ & $118.67(10)$ & $\mathrm{C} 25-\mathrm{N} 4-\mathrm{C} 29$ & $119.17(10)$ \\
\hline $\mathrm{C} 1-\mathrm{N} 2-\mathrm{C} 13$ & $117.95(10)$ & $\mathrm{C} 17-\mathrm{N} 4-\mathrm{C} 29$ & $116.80(10)$ \\
\hline $\mathrm{C} 2-\mathrm{C} 1-\mathrm{C} 6$ & $118.91(11)$ & $\mathrm{C} 18-\mathrm{C} 17-\mathrm{C} 22$ & $118.86(11)$ \\
\hline $\mathrm{C} 2-\mathrm{C} 1-\mathrm{N} 2$ & $119.06(11)$ & $\mathrm{C} 18-\mathrm{C} 17-\mathrm{N} 4$ & $118.36(11)$ \\
\hline $\mathrm{C} 6-\mathrm{C} 1-\mathrm{N} 2$ & $121.94(10)$ & $\mathrm{C} 22-\mathrm{C} 17-\mathrm{N} 4$ & $122.65(10)$ \\
\hline $\mathrm{C} 3-\mathrm{C} 2-\mathrm{C} 1$ & $120.99(12)$ & $\mathrm{C} 19-\mathrm{C} 18-\mathrm{C} 17$ & $121.09(12)$ \\
\hline $\mathrm{C} 3-\mathrm{C} 2-\mathrm{H} 2$ & $121.3(8)$ & $\mathrm{C} 19-\mathrm{C} 18-\mathrm{H} 18$ & $120.4(8)$ \\
\hline $\mathrm{C} 1-\mathrm{C} 2-\mathrm{H} 2$ & $117.7(8)$ & $\mathrm{C} 17-\mathrm{C} 18-\mathrm{H} 18$ & $118.5(8)$ \\
\hline
\end{tabular}




\begin{tabular}{|c|c|c|c|}
\hline $\mathrm{C} 2-\mathrm{C} 3-\mathrm{C} 4$ & $119.77(12)$ & $\mathrm{C} 18-\mathrm{C} 19-\mathrm{C} 20$ & $119.57(12)$ \\
\hline $\mathrm{C} 2-\mathrm{C} 3-\mathrm{H} 3$ & $119.3(8)$ & $\mathrm{C} 18-\mathrm{C} 19-\mathrm{H} 19$ & $119.0(8)$ \\
\hline $\mathrm{C} 4-\mathrm{C} 3-\mathrm{H} 3$ & $121.0(8)$ & $\mathrm{C} 20-\mathrm{C} 19-\mathrm{H} 19$ & $121.4(8)$ \\
\hline $\mathrm{C} 5-\mathrm{C} 4-\mathrm{C} 3$ & $120.16(12)$ & $\mathrm{C} 21-\mathrm{C} 20-\mathrm{C} 19$ & $120.30(12)$ \\
\hline $\mathrm{C} 5-\mathrm{C} 4-\mathrm{H} 4$ & $119.6(9)$ & $\mathrm{C} 21-\mathrm{C} 20-\mathrm{H} 20$ & $118.9(9)$ \\
\hline $\mathrm{C} 3-\mathrm{C} 4-\mathrm{H} 4$ & $120.2(9)$ & $\mathrm{C} 19-\mathrm{C} 20-\mathrm{H} 20$ & $120.7(9)$ \\
\hline $\mathrm{C} 4-\mathrm{C} 5-\mathrm{C} 6$ & $120.43(13)$ & $\mathrm{C} 20-\mathrm{C} 21-\mathrm{C} 22$ & $120.39(13)$ \\
\hline $\mathrm{C} 4-\mathrm{C} 5-\mathrm{H} 5$ & $122.2(8)$ & $\mathrm{C} 20-\mathrm{C} 21-\mathrm{H} 21$ & $122.3(8)$ \\
\hline $\mathrm{C} 6-\mathrm{C} 5-\mathrm{H} 5$ & $117.4(8)$ & $\mathrm{C} 22-\mathrm{C} 21-\mathrm{H} 21$ & $117.3(8)$ \\
\hline $\mathrm{C} 5-\mathrm{C} 6-\mathrm{C} 1$ & $119.73(11)$ & $\mathrm{C} 21-\mathrm{C} 22-\mathrm{C} 17$ & $119.69(11)$ \\
\hline $\mathrm{C} 5-\mathrm{C} 6-\mathrm{N} 1$ & $118.21(11)$ & $\mathrm{C} 21-\mathrm{C} 22-\mathrm{N} 3$ & $117.74(11)$ \\
\hline $\mathrm{C} 1-\mathrm{C} 6-\mathrm{N} 1$ & $122.02(11)$ & $\mathrm{C} 17-\mathrm{C} 22-\mathrm{N} 3$ & $122.54(11)$ \\
\hline $\mathrm{N} 1-\mathrm{C} 7-\mathrm{C} 10$ & $121.39(11)$ & $\mathrm{N} 3-\mathrm{C} 23-\mathrm{C} 26$ & $121.48(12)$ \\
\hline $\mathrm{N} 1-\mathrm{C} 7-\mathrm{C} 8$ & $116.55(11)$ & $\mathrm{N} 3-\mathrm{C} 23-\mathrm{C} 24$ & $116.67(11)$ \\
\hline $\mathrm{C} 10-\mathrm{C} 7-\mathrm{C} 8$ & $122.07(11)$ & $\mathrm{C} 26-\mathrm{C} 23-\mathrm{C} 24$ & $121.85(11)$ \\
\hline $\mathrm{C} 7-\mathrm{C} 8-\mathrm{C} 9$ & $109.20(11)$ & $\mathrm{C} 23-\mathrm{C} 24-\mathrm{C} 25$ & $109.99(10)$ \\
\hline $\mathrm{C} 7-\mathrm{C} 8-\mathrm{H} 8 \mathrm{~A}$ & $108.7(8)$ & $\mathrm{C} 23-\mathrm{C} 24-\mathrm{H} 24 \mathrm{~A}$ & $109.3(9)$ \\
\hline $\mathrm{C} 9-\mathrm{C} 8-\mathrm{H} 8 \mathrm{~A}$ & $110.6(9)$ & $\mathrm{C} 25-\mathrm{C} 24-\mathrm{H} 24 \mathrm{~A}$ & $106.8(9)$ \\
\hline $\mathrm{C} 7-\mathrm{C} 8-\mathrm{H} 8 \mathrm{~B}$ & $110.3(9)$ & $\mathrm{C} 23-\mathrm{C} 24-\mathrm{H} 24 \mathrm{~B}$ & $109.5(8)$ \\
\hline $\mathrm{C} 9-\mathrm{C} 8-\mathrm{H} 8 \mathrm{~B}$ & $107.5(8)$ & $\mathrm{C} 25-\mathrm{C} 24-\mathrm{H} 24 \mathrm{~B}$ & $109.8(8)$ \\
\hline $\mathrm{H} 8 \mathrm{~A}-\mathrm{C} 8-\mathrm{H} 8 \mathrm{~B}$ & $110.5(12)$ & $\mathrm{H} 24 \mathrm{~A}-\mathrm{C} 24-\mathrm{H} 24 \mathrm{~B}$ & $111.4(13)$ \\
\hline $\mathrm{O} 1-\mathrm{C} 9-\mathrm{N} 2$ & $122.40(12)$ & $\mathrm{O} 3-\mathrm{C} 25-\mathrm{N} 4$ & $122.76(11)$ \\
\hline $\mathrm{O} 1-\mathrm{C} 9-\mathrm{C} 8$ & $121.18(11)$ & $\mathrm{O} 3-\mathrm{C} 25-\mathrm{C} 24$ & $121.48(11)$ \\
\hline $\mathrm{N} 2-\mathrm{C} 9-\mathrm{C} 8$ & $116.38(11)$ & $\mathrm{N} 4-\mathrm{C} 25-\mathrm{C} 24$ & $115.73(10)$ \\
\hline $\mathrm{C} 7-\mathrm{C} 10-\mathrm{C} 11$ & $122.38(12)$ & $\mathrm{C} 23-\mathrm{C} 26-\mathrm{C} 27$ & $123.00(12)$ \\
\hline $\mathrm{C} 7-\mathrm{C} 10-\mathrm{H} 10$ & $120.3(9)$ & $\mathrm{C} 23-\mathrm{C} 26-\mathrm{H} 26$ & $118.0(8)$ \\
\hline $\mathrm{C} 11-\mathrm{C} 10-\mathrm{H} 10$ & $117.3(9)$ & $\mathrm{C} 27-\mathrm{C} 26-\mathrm{H} 26$ & $118.9(8)$ \\
\hline $\mathrm{O} 2-\mathrm{C} 11-\mathrm{C} 10$ & $122.32(11)$ & $\mathrm{O} 4-\mathrm{C} 27-\mathrm{C} 26$ & $122.69(11)$ \\
\hline $\mathrm{O} 2-\mathrm{C} 11-\mathrm{C} 12$ & $118.72(12)$ & $\mathrm{O} 4-\mathrm{C} 27-\mathrm{C} 28$ & $118.82(12)$ \\
\hline $\mathrm{C} 10-\mathrm{C} 11-\mathrm{C} 12$ & $118.95(12)$ & $\mathrm{C} 26-\mathrm{C} 27-\mathrm{C} 28$ & $118.50(12)$ \\
\hline $\mathrm{C} 11-\mathrm{C} 12-\mathrm{H} 12 \mathrm{~A}$ & $112.7(10)$ & $\mathrm{C} 27-\mathrm{C} 28-\mathrm{H} 28 \mathrm{~A}$ & 109.5 \\
\hline $\mathrm{C} 11-\mathrm{C} 12-\mathrm{H} 12 \mathrm{~B}$ & $109.2(10)$ & $\mathrm{C} 27-\mathrm{C} 28-\mathrm{H} 28 \mathrm{~B}$ & 109.5 \\
\hline $\mathrm{H} 12 \mathrm{~A}-\mathrm{C} 12-\mathrm{H} 12 \mathrm{~B}$ & $110.0(15)$ & $\mathrm{H} 28 \mathrm{~A}-\mathrm{C} 28-\mathrm{H} 28 \mathrm{~B}$ & 109.5 \\
\hline $\mathrm{C} 11-\mathrm{C} 12-\mathrm{H} 12 \mathrm{C}$ & $108.3(11)$ & $\mathrm{C} 27-\mathrm{C} 28-\mathrm{H} 28 \mathrm{C}$ & 109.5 \\
\hline $\mathrm{H} 12 \mathrm{~A}-\mathrm{C} 12-\mathrm{H} 12 \mathrm{C}$ & $107.4(14)$ & $\mathrm{H} 28 \mathrm{~A}-\mathrm{C} 28-\mathrm{H} 28 \mathrm{C}$ & 109.5 \\
\hline $\mathrm{H} 12 \mathrm{~B}-\mathrm{C} 12-\mathrm{H} 12 \mathrm{C}$ & $109.1(15)$ & $\mathrm{H} 28 \mathrm{~B}-\mathrm{C} 28-\mathrm{H} 28 \mathrm{C}$ & 109.5 \\
\hline $\mathrm{N} 2-\mathrm{C} 13-\mathrm{C} 14$ & $112.25(11)$ & $\mathrm{N} 4-\mathrm{C} 29-\mathrm{C} 30$ & $111.51(10)$ \\
\hline $\mathrm{N} 2-\mathrm{C} 13-\mathrm{H} 13 \mathrm{~A}$ & $110.0(10)$ & $\mathrm{N} 4-\mathrm{C} 29-\mathrm{H} 29 \mathrm{~A}$ & $106.9(8)$ \\
\hline $\mathrm{C} 14-\mathrm{C} 13-\mathrm{H} 13 \mathrm{~A}$ & $112.2(10)$ & $\mathrm{C} 30-\mathrm{C} 29-\mathrm{H} 29 \mathrm{~A}$ & $110.2(8)$ \\
\hline $\mathrm{N} 2-\mathrm{C} 13-\mathrm{H} 13 \mathrm{~B}$ & $105.2(8)$ & $\mathrm{N} 4-\mathrm{C} 29-\mathrm{H} 29 \mathrm{~B}$ & $111.1(8)$ \\
\hline $\mathrm{C} 14-\mathrm{C} 13-\mathrm{H} 13 \mathrm{~B}$ & $110.1(8)$ & $\mathrm{C} 30-\mathrm{C} 29-\mathrm{H} 29 \mathrm{~B}$ & $108.8(8)$ \\
\hline $\mathrm{H} 13 \mathrm{~A}-\mathrm{C} 13-\mathrm{H} 13 \mathrm{~B}$ & $106.6(12)$ & $\mathrm{H} 29 \mathrm{~A}-\mathrm{C} 29-\mathrm{H} 29 \mathrm{~B}$ & $108.2(11)$ \\
\hline $\mathrm{C} 13-\mathrm{C} 14-\mathrm{C} 15$ & $114.98(12)$ & $\mathrm{C} 29-\mathrm{C} 30-\mathrm{C} 31$ & $113.61(11)$ \\
\hline $\mathrm{C} 13-\mathrm{C} 14-\mathrm{H} 14 \mathrm{~A}$ & $110.6(9)$ & $\mathrm{C} 29-\mathrm{C} 30-\mathrm{H} 30 \mathrm{~A}$ & $108.1(9)$ \\
\hline $\mathrm{C} 15-\mathrm{C} 14-\mathrm{H} 14 \mathrm{~A}$ & $107.9(9)$ & $\mathrm{C} 31-\mathrm{C} 30-\mathrm{H} 30 \mathrm{~A}$ & $108.5(8)$ \\
\hline $\mathrm{C} 13-\mathrm{C} 14-\mathrm{H} 14 \mathrm{~B}$ & $107.6(9)$ & $\mathrm{C} 29-\mathrm{C} 30-\mathrm{H} 30 \mathrm{~B}$ & $108.9(8)$ \\
\hline $\mathrm{C} 15-\mathrm{C} 14-\mathrm{H} 14 \mathrm{~B}$ & $109.0(9)$ & $\mathrm{C} 31-\mathrm{C} 30-\mathrm{H} 30 \mathrm{~B}$ & $112.1(8)$ \\
\hline $\mathrm{H} 14 \mathrm{~A}-\mathrm{C} 14-\mathrm{H} 14 \mathrm{~B}$ & $106.4(13)$ & $\mathrm{H} 30 \mathrm{~A}-\mathrm{C} 30-\mathrm{H} 30 \mathrm{~B}$ & $105.2(11)$ \\
\hline
\end{tabular}




\begin{tabular}{|c|c|}
\hline $\mathrm{C} 16-\mathrm{C} 15-\mathrm{C} 14$ & $112.47(13)$ \\
\hline $\mathrm{C} 16-\mathrm{C} 15-\mathrm{H} 15 \mathrm{~A}$ & $108.2(10)$ \\
\hline $\mathrm{C} 14-\mathrm{C} 15-\mathrm{H} 15 \mathrm{~A}$ & $109.5(10)$ \\
\hline $\mathrm{C} 16-\mathrm{C} 15-\mathrm{H} 15 \mathrm{~B}$ & $111.8(9)$ \\
\hline $\mathrm{C} 14-\mathrm{C} 15-\mathrm{H} 15 \mathrm{~B}$ & $108.1(9)$ \\
\hline $\mathrm{H} 15 \mathrm{~A}-\mathrm{C} 15-\mathrm{H} 15 \mathrm{~B}$ & $106.6(14)$ \\
\hline $\mathrm{C} 15-\mathrm{C} 16-\mathrm{H} 16 \mathrm{~A}$ & $111.0(11)$ \\
\hline $\mathrm{C} 15-\mathrm{C} 16-\mathrm{H} 16 \mathrm{~B}$ & $113.0(11)$ \\
\hline $\mathrm{H} 16 \mathrm{~A}-\mathrm{C} 16-\mathrm{H} 16 \mathrm{~B}$ & $107.2(16)$ \\
\hline $\mathrm{C} 15-\mathrm{C} 16-\mathrm{H} 16 \mathrm{C}$ & $112.3(11)$ \\
\hline $\mathrm{H} 16 \mathrm{~A}-\mathrm{C} 16-\mathrm{H} 16 \mathrm{C}$ & $107.2(14)$ \\
\hline $\mathrm{H} 16 \mathrm{~B}-\mathrm{C} 16-\mathrm{H} 16 \mathrm{C}$ & $105.7(14)$ \\
\hline $\mathrm{C} 9-\mathrm{N} 2-\mathrm{C} 1-\mathrm{C} 2$ & $-131.39(13)$ \\
\hline $\mathrm{C} 13-\mathrm{N} 2-\mathrm{C} 1-\mathrm{C} 2$ & $46.95(15)$ \\
\hline $\mathrm{C} 9-\mathrm{N} 2-\mathrm{C} 1-\mathrm{C} 6$ & $52.13(16)$ \\
\hline $\mathrm{C} 13-\mathrm{N} 2-\mathrm{C} 1-\mathrm{C} 6$ & $-129.53(13)$ \\
\hline $\mathrm{C} 6-\mathrm{C} 1-\mathrm{C} 2-\mathrm{C} 3$ & $0.67(19)$ \\
\hline $\mathrm{N} 2-\mathrm{C} 1-\mathrm{C} 2-\mathrm{C} 3$ & $-175.92(11)$ \\
\hline $\mathrm{C} 1-\mathrm{C} 2-\mathrm{C} 3-\mathrm{C} 4$ & $0.34(19)$ \\
\hline $\mathrm{C} 2-\mathrm{C} 3-\mathrm{C} 4-\mathrm{C} 5$ & $-0.78(19)$ \\
\hline $\mathrm{C} 3-\mathrm{C} 4-\mathrm{C} 5-\mathrm{C} 6$ & $0.20(19)$ \\
\hline $\mathrm{C} 4-\mathrm{C} 5-\mathrm{C} 6-\mathrm{C} 1$ & $0.82(19)$ \\
\hline $\mathrm{C} 4-\mathrm{C} 5-\mathrm{C} 6-\mathrm{N} 1$ & $178.38(12)$ \\
\hline $\mathrm{C} 2-\mathrm{C} 1-\mathrm{C} 6-\mathrm{C} 5$ & $-1.24(18)$ \\
\hline $\mathrm{N} 2-\mathrm{C} 1-\mathrm{C} 6-\mathrm{C} 5$ & $175.25(11)$ \\
\hline $\mathrm{C} 2-\mathrm{C} 1-\mathrm{C} 6-\mathrm{N} 1$ & $-178.70(11)$ \\
\hline $\mathrm{N} 2-\mathrm{C} 1-\mathrm{C} 6-\mathrm{N} 1$ & $-2.21(18)$ \\
\hline $\mathrm{C} 7-\mathrm{N} 1-\mathrm{C} 6-\mathrm{C} 5$ & $138.62(13)$ \\
\hline $\mathrm{C} 7-\mathrm{N} 1-\mathrm{C} 6-\mathrm{C} 1$ & $-43.88(18)$ \\
\hline $\mathrm{C} 6-\mathrm{N} 1-\mathrm{C} 7-\mathrm{C} 10$ & $-178.93(12)$ \\
\hline $\mathrm{C} 6-\mathrm{N} 1-\mathrm{C} 7-\mathrm{C} 8$ & $1.32(18)$ \\
\hline $\mathrm{N} 1-\mathrm{C} 7-\mathrm{C} 8-\mathrm{C} 9$ & $70.83(13)$ \\
\hline $\mathrm{C} 10-\mathrm{C} 7-\mathrm{C} 8-\mathrm{C} 9$ & $-108.91(13)$ \\
\hline $\mathrm{C} 1-\mathrm{N} 2-\mathrm{C} 9-\mathrm{O} 1$ & $170.18(11)$ \\
\hline $\mathrm{C} 13-\mathrm{N} 2-\mathrm{C} 9-\mathrm{O} 1$ & $-8.15(17)$ \\
\hline $\mathrm{C} 1-\mathrm{N} 2-\mathrm{C} 9-\mathrm{C} 8$ & $-12.36(16)$ \\
\hline $\mathrm{C} 13-\mathrm{N} 2-\mathrm{C} 9-\mathrm{C} 8$ & $169.31(10)$ \\
\hline $\mathrm{C} 7-\mathrm{C} 8-\mathrm{C} 9-\mathrm{O} 1$ & $113.48(13)$ \\
\hline $\mathrm{C} 7-\mathrm{C} 8-\mathrm{C} 9-\mathrm{N} 2$ & $-64.01(13)$ \\
\hline $\mathrm{N} 1-\mathrm{C} 7-\mathrm{C} 10-\mathrm{C} 11$ & $2.17(19)$ \\
\hline $\mathrm{C} 8-\mathrm{C} 7-\mathrm{C} 10-\mathrm{C} 11$ & $-178.09(11)$ \\
\hline $\mathrm{C} 7-\mathrm{C} 10-\mathrm{C} 11-\mathrm{O} 2$ & $-0.45(19)$ \\
\hline $\mathrm{C} 7-\mathrm{C} 10-\mathrm{C} 11-\mathrm{C} 12$ & $179.61(12)$ \\
\hline $\mathrm{C} 9-\mathrm{N} 2-\mathrm{C} 13-\mathrm{C} 14$ & $-121.28(12)$ \\
\hline $\mathrm{C} 1-\mathrm{N} 2-\mathrm{C} 13-\mathrm{C} 14$ & $60.30(14)$ \\
\hline
\end{tabular}

\begin{tabular}{|c|c|}
\hline $\mathrm{C} 30-\mathrm{C} 31-\mathrm{C} 32$ & $112.70(13)$ \\
\hline $\mathrm{C} 30-\mathrm{C} 31-\mathrm{H} 31 \mathrm{~A}$ & $109.6(8)$ \\
\hline $\mathrm{C} 32-\mathrm{C} 31-\mathrm{H} 31 \mathrm{~A}$ & $110.4(9)$ \\
\hline $\mathrm{C} 30-\mathrm{C} 31-\mathrm{H} 31 \mathrm{~B}$ & $109.8(9)$ \\
\hline $\mathrm{C} 32-\mathrm{C} 31-\mathrm{H} 31 \mathrm{~B}$ & $109.4(9)$ \\
\hline $\mathrm{H} 31 \mathrm{~A}-\mathrm{C} 31-\mathrm{H} 31 \mathrm{~B}$ & $104.6(12)$ \\
\hline $\mathrm{C} 31-\mathrm{C} 32-\mathrm{H} 32 \mathrm{~A}$ & $110.7(11)$ \\
\hline $\mathrm{C} 31-\mathrm{C} 32-\mathrm{H} 32 \mathrm{~B}$ & $109.7(10)$ \\
\hline $\mathrm{H} 32 \mathrm{~A}-\mathrm{C} 32-\mathrm{H} 32 \mathrm{~B}$ & $109.6(14)$ \\
\hline $\mathrm{C} 31-\mathrm{C} 32-\mathrm{H} 32 \mathrm{C}$ & $110.6(10)$ \\
\hline $\mathrm{H} 32 \mathrm{~A}-\mathrm{C} 32-\mathrm{H} 32 \mathrm{C}$ & $109.8(14)$ \\
\hline $\mathrm{H} 32 \mathrm{~B}-\mathrm{C} 32-\mathrm{H} 32 \mathrm{C}$ & $106.4(13)$ \\
\hline $\mathrm{C} 25-\mathrm{N} 4-\mathrm{C} 17-\mathrm{C} 18$ & $-133.96(13)$ \\
\hline $\mathrm{C} 29-\mathrm{N} 4-\mathrm{C} 17-\mathrm{C} 18$ & $50.34(15)$ \\
\hline $\mathrm{C} 25-\mathrm{N} 4-\mathrm{C} 17-\mathrm{C} 22$ & $50.23(17)$ \\
\hline $\mathrm{C} 29-\mathrm{N} 4-\mathrm{C} 17-\mathrm{C} 22$ & $-125.47(13)$ \\
\hline $\mathrm{C} 22-\mathrm{C} 17-\mathrm{C} 18-\mathrm{C} 19$ & $3.05(19)$ \\
\hline $\mathrm{N} 4-\mathrm{C} 17-\mathrm{C} 18-\mathrm{C} 19$ & $-172.93(12)$ \\
\hline $\mathrm{C} 17-\mathrm{C} 18-\mathrm{C} 19-\mathrm{C} 20$ & $-0.4(2)$ \\
\hline $\mathrm{C} 18-\mathrm{C} 19-\mathrm{C} 20-\mathrm{C} 21$ & $-1.7(2)$ \\
\hline $\mathrm{C} 19-\mathrm{C} 20-\mathrm{C} 21-\mathrm{C} 22$ & $1.1(2)$ \\
\hline $\mathrm{C} 20-\mathrm{C} 21-\mathrm{C} 22-\mathrm{C} 17$ & $1.57(19)$ \\
\hline $\mathrm{C} 20-\mathrm{C} 21-\mathrm{C} 22-\mathrm{N} 3$ & $179.63(12)$ \\
\hline $\mathrm{C} 18-\mathrm{C} 17-\mathrm{C} 22-\mathrm{C} 21$ & $-3.61(18)$ \\
\hline $\mathrm{N} 4-\mathrm{C} 17-\mathrm{C} 22-\mathrm{C} 21$ & $172.18(11)$ \\
\hline $\mathrm{C} 18-\mathrm{C} 17-\mathrm{C} 22-\mathrm{N} 3$ & $178.43(12)$ \\
\hline $\mathrm{N} 4-\mathrm{C} 17-\mathrm{C} 22-\mathrm{N} 3$ & $-5.78(19)$ \\
\hline $\mathrm{C} 23-\mathrm{N} 3-\mathrm{C} 22-\mathrm{C} 21$ & $140.48(13)$ \\
\hline $\mathrm{C} 23-\mathrm{N} 3-\mathrm{C} 22-\mathrm{C} 17$ & $-41.51(19)$ \\
\hline $\mathrm{C} 22-\mathrm{N} 3-\mathrm{C} 23-\mathrm{C} 26$ & $-177.17(12)$ \\
\hline $\mathrm{C} 22-\mathrm{N} 3-\mathrm{C} 23-\mathrm{C} 24$ & $3.50(18)$ \\
\hline $\mathrm{N} 3-\mathrm{C} 23-\mathrm{C} 24-\mathrm{C} 25$ & $69.03(14)$ \\
\hline $\mathrm{C} 26-\mathrm{C} 23-\mathrm{C} 24-\mathrm{C} 25$ & $-110.29(13)$ \\
\hline $\mathrm{C} 17-\mathrm{N} 4-\mathrm{C} 25-\mathrm{O} 3$ & $175.20(11)$ \\
\hline $\mathrm{C} 29-\mathrm{N} 4-\mathrm{C} 25-\mathrm{O} 3$ & $-9.19(17)$ \\
\hline $\mathrm{C} 17-\mathrm{N} 4-\mathrm{C} 25-\mathrm{C} 24$ & $-6.60(16)$ \\
\hline $\mathrm{C} 29-\mathrm{N} 4-\mathrm{C} 25-\mathrm{C} 24$ & $169.00(10)$ \\
\hline $\mathrm{C} 23-\mathrm{C} 24-\mathrm{C} 25-\mathrm{O} 3$ & $110.58(13)$ \\
\hline $\mathrm{C} 23-\mathrm{C} 24-\mathrm{C} 25-\mathrm{N} 4$ & $-67.64(13)$ \\
\hline $\mathrm{N} 3-\mathrm{C} 23-\mathrm{C} 26-\mathrm{C} 27$ & $2.88(19)$ \\
\hline $\mathrm{C} 24-\mathrm{C} 23-\mathrm{C} 26-\mathrm{C} 27$ & $-177.82(11)$ \\
\hline $\mathrm{C} 23-\mathrm{C} 26-\mathrm{C} 27-\mathrm{O} 4$ & $3.2(2)$ \\
\hline $\mathrm{C} 23-\mathrm{C} 26-\mathrm{C} 27-\mathrm{C} 28$ & $-176.60(12)$ \\
\hline $\mathrm{C} 25-\mathrm{N} 4-\mathrm{C} 29-\mathrm{C} 30$ & $-109.33(12)$ \\
\hline $\mathrm{C} 17-\mathrm{N} 4-\mathrm{C} 29-\mathrm{C} 30$ & $66.58(13)$ \\
\hline
\end{tabular}




$\begin{array}{llll}\mathrm{N} 2-\mathrm{C} 13-\mathrm{C} 14-\mathrm{C} 15 & 55.44(16) & \mathrm{N} 4-\mathrm{C} 29-\mathrm{C} 30-\mathrm{C} 31 & 59.09(14) \\ \mathrm{C} 13-\mathrm{C} 14-\mathrm{C} 15-\mathrm{C} 16 & 177.96(13) & \mathrm{C} 29-\mathrm{C} 30-\mathrm{C} 31-\mathrm{C} 32 & 174.97(12)\end{array}$

Symmetry codes: (i) $-x+1,-y+1,-z+1$; (ii) $-x+2,-y,-z+1$; (iii) $-x+1,-y,-z+1$; (iv) $-x+1,-y+1,-z$; (v) $-x+1,-y+2,-z$; (vi) $-x+2,-y+1,-z$; (vii) $x$, $y-1, z$; (viii) $x+1, y, z$; (ix) $-x+2,-y+1,-z+1$; (x) $x-1, y, z$; (xi) $-x+2,-y+2,-z$.

Hydrogen-bond geometry $\left(\AA,{ }^{\circ}\right)$

$\mathrm{Cg} 1$ is the centroid of benzene ring $A(\mathrm{C} 1-\mathrm{C} 6)$.

\begin{tabular}{lllll}
\hline$D-\mathrm{H} \cdots A$ & $D-\mathrm{H}$ & $\mathrm{H} \cdots A$ & $D \cdots A$ & $D-\mathrm{H} \cdots A$ \\
\hline $\mathrm{N} 1-\mathrm{H} 1 \cdots \mathrm{O} 2$ & $0.927(17)$ & $1.834(17)$ & $2.5998(14)$ & $138.2(13)$ \\
$\mathrm{N} 3-\mathrm{H} 3 A \cdots \mathrm{O} 4$ & $0.898(17)$ & $1.901(17)$ & $2.6349(14)$ & $137.6(14)$ \\
$\mathrm{C} 2-\mathrm{H} 2 \cdots \mathrm{O} 1^{\mathrm{ii}}$ & $0.964(15)$ & $2.469(16)$ & $3.4235(17)$ & $170.6(11)$ \\
$\mathrm{C} 3-\mathrm{H} 3 \cdots 3^{\text {vi }}$ & $0.968(15)$ & $2.420(17)$ & $3.3714(16)$ & $166.0(11)$ \\
$\mathrm{C} 5-\mathrm{H} 5 \cdots \mathrm{O} 4$ & $0.998(16)$ & $2.456(15)$ & $3.4086(17)$ & $159.3(11)$ \\
$\mathrm{C} 18-\mathrm{H} 18 \cdots \mathrm{O} 3^{v}$ & $0.961(14)$ & $2.556(15)$ & $3.5165(16)$ & $176.4(11)$ \\
$\mathrm{C} 19-\mathrm{H} 19 \cdots \mathrm{O} 1^{\mathrm{i}}$ & $1.001(15)$ & $2.330(15)$ & $3.3273(15)$ & $177.0(12)$ \\
$\mathrm{C} 21-\mathrm{H} 21 \cdots \mathrm{O} 2$ & $0.986(15)$ & $2.277(15)$ & $3.1933(16)$ & $154.1(11)$ \\
$\mathrm{C} 28-\mathrm{H} 28 C \cdots \mathrm{O} 44^{\text {vi }}$ & 0.98 & 2.48 & $3.4342(18)$ & 164 \\
$\mathrm{C} 12-\mathrm{H} 12 A \cdots C g 1^{\mathrm{x}}$ & $0.999(19)$ & $2.921(19)$ & $3.9047(16)$ & $167.8(13)$ \\
$\mathrm{C} 30-\mathrm{H} 30 A \cdots C g 1^{\text {xii }}$ & $1.007(16)$ & $2.903(15)$ & $3.8016(15)$ & $149.0(11)$
\end{tabular}

Symmetry codes: (i) $-x+1,-y+1,-z+1$; (ii) $-x+2,-y,-z+1$; (v) $-x+1,-y+2,-z$; (vi) $-x+2,-y+1,-z$; (x) $x-1, y, z$; (xii) $x, y+1, z$. 